\title{
A LOWER BOUND FOR SPLINES ON TETRAHEDRAL VERTEX STARS
}

\author{
MICHAEL DIPASQUALE AND NELLY VILLAMIZAR
}

\begin{abstract}
A tetrahedral complex all of whose tetrahedra meet at a common vertex is called a vertex star. Vertex stars are a natural generalization of planar triangulations, and understanding splines on vertex stars is a crucial step to analyzing trivariate splines. It is particularly difficult to compute the dimension of splines on vertex stars in which the vertex is completely surrounded by tetrahedra - we call these closed vertex stars. A formula due to Alfeld, Neamtu, and Schumaker gives the dimension of $C^{r}$ splines on closed vertex stars of degree at least $3 r+2$. We show that this formula is a lower bound on the dimension of $C^{r}$ splines of degree at least $(3 r+2) / 2$. Our proof uses apolarity and the so-called Waldschmidt constant of the set of points dual to the interior faces of the vertex star. We also use an argument of Whiteley to show that the only splines of degree at most $(3 r+1) / 2$ on a generic closed vertex star are global polynomials.
\end{abstract}

\section{INTRODUCTION}

A multivariate spline is a piecewise polynomial function on a partition $\Delta$ of some domain $\Omega \subset \mathbb{R}^{n}$ which is continuously differentiable to order $r$ for some integer $r \geq 0$. Multivariate splines play an important role in many areas such as finite elements, computer-aided design, and data fitting [12, 22]. In these applications it is important to construct a basis, often with prescribed properties, for splines of bounded total degree. A more basic task which aids in the construction of a basis is simply to compute the dimension of the space of multivariate splines of bounded degree on a fixed partition. We write $\mathcal{S}_{d}^{r}(\Delta)$ for the vector space of piecewise polynomial functions of degree at most $d$ on the partition $\Delta$ which are continuously differentiable of order $r$.

A formula for the dimension of $\mathcal{S}_{d}^{1}(\Delta)$, where $\Delta$ is a planar triangulation, was first proposed by Strang [37] and proved for $d \geq 2$ by Billera for generic vertex positions [5]. Subsequently the problem of computing the dimension of planar splines on triangulations has received considerable attention using a wide variety of techniques, see [2, 3, 5, 6, 21, 32, 33, 35, 39, 40]. Hong shows in [20] that the dimension of $\mathcal{S}_{d}^{r}(\Delta)$, for $\Delta$ a planar triangulation and $d \geq 3 r+2$, is given by a quadratic polynomial in $d$ whose coefficients are determined from simple data of the triangulation. An important feature of planar splines is that the formula which gives the dimension of the spline space $\mathcal{S}_{d}^{r}(\Delta)$ for $d \geq 3 r+2$ is a lower bound for any degree $d \geq 0$ [34].

In this paper we focus on splines over the union of tetrahedra all of which meet at a common vertex. We call such a configuration a star of a vertex (this is sometimes called a cell in the approximation theory literature $[22,36])$. If $\Delta$ is the star of a vertex, every spline can be written as a sum of homogeneous splines; a homogeneous spline of degree $d$ is one which restricts to a homogeneous polynomial of degree $d$ on each tetrahedron. We denote by $\mathcal{H}_{d}^{r}(\Delta)$ the vector space of homogeneous splines of degree $d$ in $\mathcal{S}_{d}^{r}(\Delta)$. Understanding homogeneous splines on vertex stars is crucial to computing the dimension of trivariate splines on tetrahedral complexes (see [4]) - whose behavior even in large degree is a major open problem in numerical analysis. We apply our present results on vertex stars to tetrahedral splines of large degree in a forthcoming paper.

In [1], Alfeld, Neamtu, and Schumaker derive formulas for the dimension of the space of homogeneous splines on vertex stars of degree $d \geq 3 r+2$. A crucial difference from the planar case is that, for splines on tetrahedral complexes, one of the dimension formulas proved in [1] is not

1991 Mathematics Subject Classification. 65D07, 41A15, 13D02, 14C20 .

Key words and phrases. Spline functions, apolarity, fat point ideals, Waldschmidt constant . 
necessarily a lower bound on the dimension of the homogeneous spline space when $d<3 r+2$. To explain this we differentiate between two types of vertex stars. If the common vertex at which all tetrahedra meet is completely surrounded by tetrahedra (so that it is the unique interior vertex), then we call the vertex star a closed vertex star. Otherwise we call the vertex star an open vertex star. It is precisely in the case of a closed vertex star when the formula of [1] may fail to be a lower bound on the dimension of the space of homogeneous splines in degrees less than $3 r+2$. This is easy to see for $d \leq r$, when the formula of [1] is exactly twice the dimension of the homogeneous spline space (which consists only of trivial splines for $d \leq r$ i.e., splines whose polynomial pieces are all equal to the same polynomial function, we refer to these splines also as global polynomials on the given partition). For an explicit and less trivial example, the reader is invited to take a look at Section 6.1, where we consider the dimension of homogeneous splines on a generic bipyramid. According to Table 1, the generic dimension of the homogeneous spline space of smoothness $r=4$ and degree $d=5$ is 21 (the only splines are global polynomials), while the formula of [1] (recorded in the column labeled $\operatorname{LB}^{\star}(\Delta, d, r)$ ) yields 27 .

The crux of our first result, Theorem 4.7, is that the formula in [1] gives a lower bound on the dimension of the homogeneous spline space $\mathcal{H}_{d}^{r}(\Delta)$ for a closed vertex star $\Delta$ with at least six boundary vertices when $d \geq(3 r+2) / 2$ and the vertex coordinates of $\Delta$ are general enough. If $\Delta$ has only four (respectively five) boundary vertices, then we show that the formula in [1] is a lower bound when $d>2 r$ (respectively, $d>(5 r+2) / 3$ ). We give some additional details to state this result more precisely, but the majority of the details we will leave to Section 4. To a closed (respectively, open) vertex star we associate the formula $\operatorname{LB}^{\star}(\Delta, d, r)$ (respectively, $\operatorname{LB}^{\aleph}(\Delta, d, r)$ ); these are defined in Section 4 in Equations (10) (for the closed vertex star) and (11) (for the open vertex star). These formulas coincide with those in [1] under the assumption that the affine spans of triangles surrounding any edge of $\Delta$ are all distinct (see Remark 4.6). Thus for a general enough choice of vertex coordinates, it follows from [1, Theorem 3] that $\operatorname{dim} \mathcal{H}_{d}^{r}(\Delta)=\mathrm{LB}^{\star}(\Delta, d, r)$ for $d \geq 3 r+2$ if $\Delta$ is a closed vertex star and that $\operatorname{dim} \mathcal{H}_{d}^{r}(\Delta)=\operatorname{LB}^{\xi}(\Delta, d, r)$ for $d \geq 3 r+2$ if $\Delta$ is an open vertex star. It is straightforward to show that $\operatorname{LB}^{\tilde{}}(\Delta, d, r) \leq \operatorname{dim} \mathcal{H}_{d}^{r}(\Delta)$ for all $d \geq 0$ if $\Delta$ is an open vertex star. On the other hand, if $\Delta$ is a closed vertex star it is quite delicate to determine the degrees $d$ for which $\operatorname{LB}^{\star}(\Delta, d, r) \leq \mathcal{H}_{d}^{r}(\Delta)$; see [36] where a lower bound is established for homogeneous $C^{2}$ splines on vertex stars. In Theorem 4.7 we establish that $\operatorname{LB}^{\star}(\Delta, d, r) \leq \operatorname{dim} \mathcal{H}_{d}^{r}(\Delta)$ for $d \geq(3 r+2) / 2$ if $\Delta$ has at least six boundary vertices (with the same adjustments as above for four or five boundary vertices).

For closed tetrahedral vertex stars, the failure of $\operatorname{LB}^{\star}(\Delta, d, r)$ to be a lower bound for $\operatorname{dim} \mathcal{H}_{d}^{r}(\Delta)$ in low degree is elucidated by homological techniques of Billera [5] as refined by Schenck and Stillman [33]. More precisely, it follows from these techniques (in particular the Billera-SchenckStillman chain complex) that

$$
\mathrm{LB}^{\star}(\Delta, d, r)-\left(\begin{array}{c}
d+2 \\
2
\end{array}\right)+\operatorname{dim} \mathrm{J}(\gamma)_{d} \leq \operatorname{dim} \mathcal{H}_{d}^{r}(\Delta)
$$

for every $d \geq 0$, where $\mathrm{J}(\gamma)$ is an ideal generated by powers of linear forms attached to the interior vertex $\gamma$ (see Proposition 4.4). Emsalem and Iarrabino showed that the dimension of an ideal generated by powers of linear forms can be computed from the Hilbert function of a so-called ideal of fat points in $\mathbb{P}^{n}$ [14]. An ideal of fat points supported at points $P_{1}, \ldots, P_{k}$ in $\mathbb{P}^{n}$ is an ideal consisting of all polynomials which vanish to some fixed order at $P_{1}, \ldots, P_{k}$ (the order of vanishing can be different for each point). In our case, the study of $\mathrm{J}(\gamma)$ leads to the consideration of ideals of fat points in $\mathbb{P}^{2}$. The Hilbert function of an ideal of fat points in $\mathbb{P}^{2}$ is quite mysterious and is the subject of much research. See, for instance, the well-celebrated Segre-Harbourne-Gimigliano-Hirschowitz conjecture [8] about the expected Hilbert function of an ideal of fat points supported at general points in $\mathbb{P}^{2}$; in spite of many partial results it still is an open problem in algebraic geometry [30, 
Section 5]. Fortunately, we need relatively little information about the Hilbert function of this ideal of fat points to establish Theorem 4.7 - a lower bound on the so-called Waldschmidt constant [7, 38] is enough to establish $\operatorname{dim} \mathrm{J}(\gamma)_{d}=\left(\begin{array}{c}d+2 \\ 2\end{array}\right)$ if $d \geq(3 r+2) / 2$. Evidently the inequality (1) then implies $\operatorname{LB}^{\star}(\Delta, d, r) \leq \operatorname{dim} \mathcal{H}_{d}^{r}(\Delta)$ for all $d \geq(3 r+2) / 2$ (with appropriate adjustment for four or five boundary vertices).

Our second result concerns homogeneous splines of small degree. It follows from work of Whiteley [39] and Alfeld, Schumaker, and Whiteley [4] that the only homogeneous splines on a generic tetrahedral vertex star of degree at most $(3 r+1) / 2$ are global polynomials (see [4, Remark 71]). Thus, if $\Delta$ is a generic vertex star with at least six interior vertices and $d \leq(3 r+1) / 2$, the inequality (1) cannot improve on the trivial lower bound of $\left(\begin{array}{c}d+2 \\ 2\end{array}\right) \leq \operatorname{dim} \mathcal{H}_{d}^{r}(\Delta)$. We prove in Theorem 5.1 that if $\Delta$ is a generic closed vertex star with four (respectively, five) interior edges, that the only homogeneous splines of degree at most $2 r$ (respectively $(5 r+2) / 3)$ are global polynomials. It follows that if $\Delta$ is any generic vertex star with four (respectively, five) boundary vertices, the inequality (1) cannot improve on the trivial lower bound of $\left(\begin{array}{c}d+2 \\ 2\end{array}\right) \leq \operatorname{dim} \mathcal{H}_{d}^{r}(\Delta)$ in degrees $d \leq 2 r$ (respectively $d \leq(5 r+2) / 3)$. This has a satisfying completeness: the formulas of [1] may not be a lower bound on $\operatorname{dim} \mathcal{H}_{d}^{r}(\Delta)$ for closed vertex stars in small degrees; however for most vertex positions there will only be trivial splines in these small degrees anyway! Even if vertex positions are not generic, it may be possible to find lower bounds whose performance is better than (1) in low degree - see Section 6.2.

The paper is organized as follows. In Section 2 we set up notation and briefly describe the homological machinery from $[5,33]$. In Section 3 we use apolarity and the Waldschmidt constant to show that $\operatorname{dim} \mathrm{J}(\gamma)_{d}=\left(\begin{array}{c}d+2 \\ 2\end{array}\right)$ if $d \geq(3 r+2) / 2$ and $\Delta$ has at least six boundary vertices (we prove corresponding statements when $\Delta$ has four or five boundary vertices). In Section 4 we prove Theorem 4.7, and in Section 5 we prove Theorem 5.1. Section 6 is devoted to illustrating our bounds in some examples and Section 7 contains concluding remarks.

\section{Background And Homological Methods}

In this section we review the necessary results from [5] and [33]. We denote by $\Delta$ a simplicial complex embedded in $\mathbb{R}^{n}$ (see [42] for basics on simplicial complexes). If $n=2$ we will refer to $\Delta$ as a triangulation, and as a tetrahedral complex if $n=3$. We denote by $\Delta_{i}^{\circ}$ the set of interior faces of $\Delta$ of dimension $i$, and by $f_{i}^{\circ}$ the number of such faces for $i=0,1, \ldots, n$. If $\beta \in \Delta_{i}$ we call $\beta$ an $i$-face. By an abuse of notation, we will identify $\Delta$ with its underlying space $\bigcup\{\beta: \beta \in \Delta\} \subset \mathbb{R}^{n}$.

Recall that a simplicial complex $\Delta$ is said to be pure if all maximal simplices have the same dimension. A pure $n$-dimensional simplicial complex $\Delta$ is hereditary if, whenever two maximal simplices $\iota, \iota^{\prime} \in \Delta_{n}$ intersect in a vertex $\gamma \in \Delta_{0}$, then there is a sequence $\iota=\iota_{1}, \iota_{2}, \ldots, \iota_{k}=\iota^{\prime}$ of $n$-dimensional simplices satisfying that $\gamma \in \iota_{i}$ for $i=1, \ldots, k$ and $\iota_{i+1} \cap \iota_{i} \in \Delta_{n-1}$ for $i=1, \ldots, k-1$.

If $\Delta$ is a pure $n$-dimensional simplicial complex all of whose $n$-dimensional simplices share a common vertex $\gamma$ then we call $\Delta$ the star of a vertex or a vertex star. Without loss of generality, we assume that $\gamma$ is at the origin. If $\gamma$ is an interior vertex of $\Delta$ then we call $\Delta$ a closed vertex star; if $\gamma$ is on the boundary of $\Delta$ then we call $\Delta$ an open vertex star. The link of a pure $n$-dimensional vertex star in which all $n$-dimensional simplices share the vertex $\gamma$ is the subcomplex of $\Delta$ consisting of all simplices of $\Delta$ which do not contain $\gamma$ (it is a subcomplex of $\Delta$ of dimension $n-1$ ). Throughout this article, whenever we refer to a simplicial complex $\Delta$, we will mean a pure, hereditary, 3-dimensional vertex star whose link is simply connected. We call these tetrahedral vertex stars, simplicial vertex stars, or simply vertex stars.

We write $\mathbf{S}=\mathbb{R}[x, y, z]$ for the polynomial ring in three variables, $\mathbf{S}_{d}$ for the vector space of homogeneous polynomials of degree $d$, and $\mathbf{S}_{\leq d}$ for the vector space of polynomials of total degree at most $d$. For a given integer $r \geq 0$, we denote by $C^{r}(\Delta)$ the set of all functions $F: \Delta \rightarrow \mathbb{R}$ which are continuously differentiable of order $r$. 
Definition 2.1. Let $\Delta \subset \mathbb{R}^{3}$ be a tetrahedral vertex star. The space $\mathcal{S}^{r}(\Delta)$ of splines on $\Delta$ is the set of piecewise polynomial functions on $\Delta$ that are continuously differentiable up to order $r$ on $\Delta$ i.e.,

$$
\mathcal{S}^{r}(\Delta)=\left\{F \in C^{r}(\Delta):\left.F\right|_{\iota} \in \mathrm{S} \text { for all } \iota \in \Delta_{3}\right\} .
$$

If we consider polynomials of degree at most $d$, the space will be denoted by $\mathcal{S}_{d}^{r}(\Delta)$, namely

$$
\mathcal{S}_{d}^{r}(\Delta)=\left\{F \in C^{r}(\Delta):\left.F\right|_{\iota} \in \mathrm{S}_{\leq d} \text { for all } \iota \in \Delta_{3}\right\}
$$

Similarly, the space $\mathcal{H}_{d}^{r}(\Delta)$ of splines whose polynomial pieces are of degree exactly $d$ is defined as

$$
\mathcal{H}_{d}^{r}(\Delta)=\left\{F \in C^{r}(\Delta):\left.F\right|_{\iota} \in \mathrm{S}_{d} \text { for all } \iota \in \Delta_{3}\right\} .
$$

The space $\mathcal{S}^{r}(\Delta)$ is itself a ring under pointwise multiplication, and the polynomial ring $\mathrm{S}$ embeds naturally into $\mathcal{S}^{r}(\Delta)$, it can be seen as the set of trivial splines or global polynomials on $\Delta$ i.e., the splines $F \in \mathcal{S}^{r}(\Delta)$ such that there exists an $f \in \mathrm{S}$ so that $\left.F\right|_{\iota}=f$ for all $\iota \in \Delta_{3}$. In this way $\mathcal{S}^{r}(\Delta)$ is both an S-module and an S-algebra. We will be concerned exclusively with the structure of $\mathcal{S}^{r}(\Delta)$ as an $\mathbb{R}$-vector space; however we may at times refer to the S-module structure of $\mathcal{S}^{r}(\Delta)$. In particular, if $\Delta$ is the star of a vertex, then it is known that

$$
\mathcal{S}^{r}(\Delta) \cong \bigoplus_{i \geq 0} \mathcal{H}_{i}^{r}(\Delta) \quad \text { and } \quad \mathcal{S}_{d}^{r}(\Delta) \cong \bigoplus_{i=0}^{d} \mathcal{H}_{i}^{r}(\Delta)
$$

where the isomorphism is as $\mathbb{R}$-vector spaces. If $F \in \mathcal{H}_{d}^{r}(\Delta)$ and $G \in \mathrm{S}_{j}$, notice that $F G \in \mathcal{H}_{d+j}^{r}(\Delta)$. This means that $\mathcal{S}_{d}^{r}(\Delta)$ has the structure of a graded S-module.

Remark 2.2. If $\Delta$ has more than one interior vertex, there is a coning construction under which the isomorphisms (2) will still be valid. As we focus on the case of vertex stars, we will not need this.

Definition 2.3. Suppose $\Delta \subset \mathbb{R}^{3}$ is a tetrahedral vertex star. If $\sigma \in \Delta_{2}$, let $\ell_{\sigma}$ be a choice of linear form vanishing on $\sigma$. We define $\mathrm{J}(\sigma)=\left\langle\ell_{\sigma}^{r+1}\right\rangle$, the ideal generated by $\ell_{\sigma}^{r+1}$. For any face $\beta \in \Delta_{i}$ where $i=0,1$ we define

$$
\mathrm{J}(\beta):=\sum_{\sigma \supseteq \beta} \mathrm{J}(\sigma)=\left\langle\ell_{\sigma}^{r+1}: \beta \subseteq \sigma\right\rangle .
$$

If $\beta \in \Delta_{3}$ we define $\mathrm{J}(\beta)=0$.

Proposition 2.4. [6, Proposition 1.2] If $\Delta$ is hereditary then $F \in \mathcal{S}^{r}(\Delta)$ if and only if

$$
\left.F\right|_{\iota}-\left.F\right|_{\iota^{\prime}} \in \mathrm{J}(\sigma) \text { for every } \iota, \iota^{\prime} \in \Delta_{3} \text { satisfying } \iota \cap \iota^{\prime}=\sigma \in \Delta_{2} \text {. }
$$

We define a chain complex introduced by Billera [5] and refined by Schenck and Stillman [33], referring the reader to [19] for undefined terms from algebraic topology. We denote the simplicial chain complex of $\Delta$ relative to its boundary $\partial \Delta$ with coefficients in $\mathrm{S}$ by $\mathcal{R}$ :

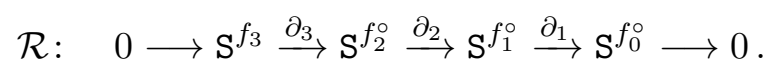

The ideals $\mathrm{J}(\beta)$ fit together to make a sub-chain complex of $\mathcal{R}$ (the differential is the restriction of the differential of $\mathcal{R}$ ):

$$
\mathcal{J}: \quad 0 \longrightarrow \bigoplus_{\sigma \in \Delta_{2}^{\circ}} \mathrm{J}(\sigma) \stackrel{\partial_{2}}{\longrightarrow} \bigoplus_{\tau \in \Delta_{1}^{\circ}} \mathrm{J}(\tau) \stackrel{\partial_{1}}{\longrightarrow} \bigoplus_{\gamma \in \Delta_{0}^{\circ}} \mathrm{J}(\gamma) \longrightarrow 0 .
$$

The Billera-Schenck-Stillman chain complex is the quotient of $\mathcal{R}$ by $\mathcal{J}$, namely

$$
\mathcal{R} / \mathcal{J}: \quad 0 \longrightarrow \bigoplus_{\iota \in \Delta_{3}} \mathrm{~S} \stackrel{\bar{\partial}_{3}}{\longrightarrow} \bigoplus_{\sigma \in \Delta_{2}^{\circ}} \frac{\mathrm{S}}{\mathrm{J}(\sigma)} \stackrel{\bar{\partial}_{2}}{\longrightarrow} \bigoplus_{\tau \in \Delta_{1}^{\circ}} \frac{\mathrm{S}}{\mathrm{J}(\tau)} \stackrel{\bar{\partial}_{1}}{\longrightarrow} \bigoplus_{\gamma \in \Delta_{0}^{\circ}} \frac{\mathrm{S}}{\mathrm{J}(\gamma)} \longrightarrow 0 .
$$


These three chain complexes fit into the evident short exact sequence of chain complexes

$$
0 \longrightarrow \mathcal{J} \longrightarrow \mathcal{R} \longrightarrow \mathcal{R} / \mathcal{J} \longrightarrow 0 \text {. }
$$

As is standard notation, $\mathcal{R}_{i}, \mathcal{J}_{i}$, and $(\mathcal{R} / \mathcal{J})_{i}$ refer to the modules in the chain complex at homological position $i$. For instance, $\mathcal{R}_{0}=\mathrm{S}^{f_{0}^{\circ}}, \mathcal{R}_{1}=\mathrm{S}^{f_{1}^{\circ}}$, and so on. We summarize some well-known properties of $\mathcal{R} / \mathcal{J}$ (see $[28,33])$.

Proposition 2.5. If $\Delta \subset \mathbb{R}^{3}$ is a tetrahedral vertex star whose link is simply connected, then $\mathcal{S}^{r}(\Delta) \cong H_{3}(\mathcal{R} / \mathcal{J}) \cong \mathrm{S} \oplus H_{2}(\mathcal{J}), H_{i}(\mathcal{R} / \mathcal{J}) \cong H_{i-1}(\mathcal{J})$ for $i=1,2$, and $H_{0}(\mathcal{R} / \mathcal{J})=0$.

The inclusion of S into $\mathcal{S}^{r}(\Delta)$ as globally polynomial corresponds (via the isomorphism in Proposition 2.5) to the copy of $\mathrm{S}$ in $\mathrm{S} \oplus H_{2}(\mathcal{J})$, while the map

$$
\bigoplus_{\sigma \in \Delta_{2}^{\circ}} \mathrm{J}(\sigma) \stackrel{\partial_{2}}{\longrightarrow} \bigoplus_{\tau \in \Delta_{1}^{\circ}} \mathrm{J}(\beta)
$$

encodes the so-called smoothing cofactors. By Proposition 2.5, the Billera-Schenck-Stillman chain complex $\mathcal{R} / \mathcal{J}$ and the chain complex $\mathcal{J}$ contain essentially the same information.

We now put everything together to write the dimension of $\mathcal{H}_{d}^{r}(\Delta)$ in terms of the Billera-SchenckStillman chain complex. If $\mathcal{C}: 0 \rightarrow C_{n} \rightarrow \cdots \rightarrow C_{0} \rightarrow 0$ is a chain complex of graded S-modules, we write $\chi(\mathcal{C}, d)$ for the graded Euler-Poincaré characteristic of $\mathcal{C}$. That is,

$$
\chi(\mathcal{C}, d)=\sum_{i=0}^{n}(-1)^{n-i} \operatorname{dim}\left(C_{i}\right)_{d} .
$$

Proposition 2.6. If $\Delta$ is a tetrahedral vertex star then

$$
\operatorname{dim} \mathcal{H}_{d}^{r}(\Delta)=\chi(\mathcal{R} / \mathcal{J}, d)+\operatorname{dim} H_{2}(\mathcal{R} / \mathcal{J})_{d}=\operatorname{dim} \mathrm{S}_{d}+\chi(\mathcal{J}, d)+\operatorname{dim} H_{1}(\mathcal{J})_{d}
$$

In particular,

$$
\operatorname{dim} \mathcal{H}_{d}^{r}(\Delta) \geq \chi(\mathcal{R} / \mathcal{J}, d)=\operatorname{dim} \mathrm{S}_{d}+\chi(\mathcal{J}, d)
$$

Proof. The equality between the expressions involving $\chi(\mathcal{R} / \mathcal{J}, d)$ and those involving $\chi(\mathcal{J}, d)$ follows immediately from Proposition 2.5. We prove the statements which involve $\mathcal{J}$, using the fact that $\chi(\mathcal{J}, d)=\sum_{i=0}^{2}(-1)^{2-i} \operatorname{dim} H_{i}(\mathcal{J})_{d}$. If $\Delta$ is a closed vertex star with interior vertex $\gamma$, then $\mathcal{J}$ has the form

$$
\mathcal{J}: \quad 0 \rightarrow \bigoplus_{\sigma \in \Delta_{2}^{\circ}} \mathrm{J}(\sigma) \stackrel{\partial_{2}}{\longrightarrow} \bigoplus_{\tau \in \Delta_{1}^{\circ}} \mathrm{J}(\tau) \stackrel{\partial_{1}}{\rightarrow} \mathrm{J}(\gamma) \rightarrow 0
$$

the map $\partial_{1}$ is surjective from the definition of $\mathrm{J}(\gamma)$, hence $H_{0}(\mathcal{J})=0$. Thus

$$
\operatorname{dim} H_{2}(\mathcal{J})_{d}-\operatorname{dim} H_{1}(\mathcal{J})_{d}=\chi(\mathcal{J}, d) .
$$

The result follows since $\operatorname{dim} \mathcal{H}_{d}^{r}(\Delta)=\operatorname{dim} \mathrm{S}_{d}+\operatorname{dim} H_{2}(\mathcal{J})_{d}$ by Proposition 2.5.

If $\Delta$ is an open vertex star, then $\mathcal{J}$ has the form

$$
\mathcal{J}: \quad 0 \rightarrow \bigoplus_{\sigma \in \Delta_{2}^{\circ}} \mathrm{J}(\sigma) \stackrel{\partial_{2}}{\longrightarrow} \bigoplus_{\tau \in \Delta_{1}^{\circ}} \mathrm{J}(\tau) \stackrel{\partial_{1}}{\rightarrow} 0 \rightarrow 0,
$$

so there is not even a vector space in homological index 0 , thus $H_{0}(\mathcal{J})=0$ as well and the formula follows from the above argument immediately.

Finally, we clarify what we mean by a generic vertex star; this is fairly standard in the literature on splines $[1,4,5,39,40]$. The main point is that it will suffice to prove the lower bound in Theorem 4.7 when $\Delta$ is a generic tetrahedral vertex star. 
Definition 2.7. Suppose $\Delta \subset \mathbb{R}^{3}$ is a star of the vertex $\gamma$. We call $\Delta$ generic with respect to a fixed $r, d$ if, for all sufficiently small perturbations of the vertices $\gamma^{\prime} \neq \gamma \in \Delta_{0}$, the resulting vertex star $\Delta^{\prime}$ satisfies $\operatorname{dim} \mathcal{H}_{d}^{r}\left(\Delta^{\prime}\right)=\operatorname{dim} \mathcal{H}_{d}^{r}(\Delta)$. If $r$ and $d$ are understood from context, we simply say $\Delta$ is generic.

Lemma 2.8. Suppose $\Delta \subset \mathbb{R}^{3}$ is a star of the vertex $\gamma$, and fix non-negative integers $r$ and $d$. Then almost all sufficiently small perturbations of the vertices $\gamma^{\prime} \neq \gamma \in \Delta_{0}$ result in a vertex star $\Delta^{\prime}$ which is generic with respect to $r$ and $d$. Moreover $\operatorname{dim} \mathcal{H}_{d}^{r}(\Delta) \geq \operatorname{dim} \mathcal{H}_{d}^{r}\left(\Delta^{\prime}\right)$.

Proof. This follows immediately from examining rank conditions on any of the equivalent ways of defining splines as the kernel of a linear transformation.

\section{Duality: fat POINTS AND POWERS OF LINEAR FORMS}

In this section we review a duality between ideals generated by powers of linear forms and ideals of polynomials which vanish to certain orders on sets of points in projective space, called fat point ideals. We reduce the presentation to our case i.e., ideals in the polynomial ring of three variables and the corresponding fat points ideals in $\mathbb{P}^{2}$. We use this duality, along with combinatorial bounds from [11], to prove Corollary 3.17, the main result of this section. Corollary 3.17 provides explicit lower bounds for the degree in which $\mathrm{J}(\gamma)_{d}=\mathrm{S}_{d}$, where $\gamma$ is the interior vertex of a closed vertex star.

We write $[a: b: c]$ for a point in projective 2 -space over $\mathbb{R}$, which we denote by $\mathbb{P}^{2}$. We let $\mathrm{R}:=\mathbb{R}[X, Y, Z]$ be the polynomial ring in three variables. If $P=[a: b: c] \in \mathbb{P}^{2}$ we write $\wp_{P}$ for the ideal of homogeneous polynomials in $\mathrm{R}$ which vanish at $P$; i.e. $\wp_{P}=\langle b X-a Y, c X-a Z, c Y-b Z\rangle$. It is straightforward to see that $\wp_{P}^{m}$ consists of all polynomials whose homogeneous components vanish to order $m$ at $P$.

Definition 3.1. Let $\mathcal{X}=\left\{P_{1}, \ldots, P_{k}\right\}$ be a collection of points in $\mathbb{P}^{2}$ and $\boldsymbol{m}=\left\{m_{1}, \ldots, m_{k}\right\}$ a collection of positive integers attached to $P_{1}, \ldots, P_{k}$, respectively. The ideal of fat points associated to $\mathcal{X}$ and $\boldsymbol{m}$ is

$$
\mathrm{I}=\mathrm{I}_{\mathcal{X}}^{\boldsymbol{m}}:=\bigcap_{i=1}^{k} \wp_{i}^{m_{i}} .
$$

If there is a positive integer $s$ so that $m_{i}=s$ for $i=1, \ldots, k$ then we write $\mathrm{I}_{\mathcal{X}}^{(s)}$ instead of $\mathrm{I}_{\mathcal{X}}^{\boldsymbol{m}}$. If $m_{i}=1$ for $i=1, \ldots, k$ we simply write $\mathrm{I}_{\mathcal{X}}$.

Remark 3.2. It is straightforward to see that $I_{\mathcal{X}}^{m}$ is the set of polynomials whose homogeneous components vanish to order $m_{i}$ at the point $P_{i}$, for $i=1, \ldots, k$. Since $\wp_{i}^{m_{i}}$ is graded for each $\sigma \in \Omega$, $\mathrm{I}_{\mathcal{X}}^{\boldsymbol{m}}$ is also a graded ideal.

Remark 3.3. The ideal $\mathrm{I}_{\mathcal{X}}^{(s)}$ in Definition 3.1 is called the sth symbolic power of $\mathrm{I}_{\mathcal{X}}$.

Now consider simultaneously the polynomial rings $\mathrm{S}=\mathbb{R}[x, y, z]$ and $\mathrm{R}=\mathbb{R}[X, Y, Z]$, and let $\mathrm{R}$ act on $\mathrm{S}$ as polynomial differential operators. Namely, if $h \in \mathrm{R}$ and $f \in \mathrm{S}$ then $h \circ f=h\left(\frac{\partial}{\partial x}, \frac{\partial}{\partial y}, \frac{\partial}{\partial z}\right) \circ f$. We call this the apolarity action of $\mathrm{R}$ on $\mathrm{S}$. The apolarity action induces a perfect pairing $\mathrm{R}_{d} \times \mathrm{S}_{d} \rightarrow \mathbb{R}$ by $(h, f) \rightarrow h \circ f$.

Example 3.4. Let $F=X^{2}+Y^{2}+Z^{2} \in \mathrm{R}$. If $f \in \mathrm{S}$, then $F \circ f=\frac{\partial^{2} f}{\partial x^{2}}+\frac{\partial^{2} f}{\partial y^{2}}+\frac{\partial^{2} f}{\partial z^{2}}$.

If $\mathrm{I} \subset \mathrm{R}$ is an ideal of $\mathrm{R}$, then the inverse system $\mathrm{I}^{\perp}$ of $\mathrm{I}$ is defined as

$$
\mathrm{I}^{\perp}:=\{f \in \mathrm{S}: h \circ f=0 \text { for all } h \in \mathrm{I}\} .
$$

If $\mathrm{I}$ is graded, then $\mathrm{I}^{\perp}$ is a graded vector space (it is generally not an ideal) with graded structure $\mathrm{I}^{\perp} \cong \bigoplus_{d \geq 0} \mathrm{I}_{d}^{\perp}$, where $\mathrm{I}_{d}^{\perp}$ is the vector space of all homogeneous polynomials of degree $d$ in $\mathrm{I}^{\perp}$. 
Example 3.5. Let $P=[0: 0: 1] \in \mathbb{P}^{2}$, with $\wp_{P}=\langle X, Y\rangle$. Then $\wp_{P}^{\perp}=\operatorname{span}\left\{1, z, z^{2}, z^{3}, \ldots\right\}$. More generally, if $P=[a: b: c]$ then $\wp_{P}^{\perp}=\operatorname{span}\left\{1, a x+b y+c z,(a x+b y+c z)^{2}, \ldots\right\}$.

For a graded ideal $\mathrm{I} \subset \mathrm{R}$, the apolarity action induces an isomorphism of vector spaces $(\mathrm{R} / \mathrm{I})_{d} \cong$ $\mathrm{I}_{d}^{\perp}$ (this follows since the apolarity action induces a perfect pairing $\mathrm{R}_{d} \times \mathrm{S}_{d} \rightarrow \mathbb{R}$ ). Thus one can deduce $\operatorname{dim} \mathrm{I}_{d}^{\perp}$ from $\operatorname{dim} \mathrm{I}_{d}$, and vice-versa. The following result of Emsalem and Iarrobino [14] describes the inverse system of a fat point ideal.

We first introduce some notation which suits our context. If $\sigma$ is a two-simplex in $\mathbb{R}^{3}$ whose affine span contains the origin, let $\ell_{\sigma}=a x+b y+c z$ be a choice of linear form vanishing on $\sigma$ (well-defined up to constant multiple). The coefficients of $\ell_{\sigma}$ define the point $P_{\sigma}=[a: b: c] \in \mathbb{P}^{2}$ (notice this point does not depend on the multiple of $\ell_{\sigma}$ chosen), which in turn defines the ideal $\wp_{\sigma}=\wp_{P_{\sigma}} \subseteq \mathrm{R}$.

Theorem 3.6 (Emsalem and Iarrabino [14]). Let $\Omega$ be a collection of two-simplices in $\mathbb{R}^{3}$ each of whose affine span contains the origin and let $m_{\sigma}$ be a positive integer attached to each $\sigma \in \Omega$. Put $\mathcal{X}=\left\{P_{\sigma}\right\}_{\sigma \in \Omega}$. If $d \geq \max \left\{m_{\sigma}+1\right\}$, let $d-\boldsymbol{m}=\left\{d-m_{\sigma}\right\}_{\sigma \in \Omega}$ and $\mathrm{I}=\mathrm{I}_{\mathcal{X}}^{d-\boldsymbol{m}}$. Then

$$
\left\langle\ell_{\sigma}^{m_{\sigma}+1}: \sigma \in \Omega\right\rangle_{d}= \begin{cases}0 & \text { for } d \leq \max \left\{m_{\sigma}\right\} \\ \left(\mathrm{I}^{\perp}\right)_{d} \cong\left(\frac{\mathrm{R}}{\mathrm{I}}\right)_{d} & \text { for } d \geq \max \left\{m_{\sigma}+1\right\} .\end{cases}
$$

Theorem 3.6 has an especially nice formulation in the case of uniform powers. We state this for the ideal $\mathrm{J}(\gamma)$ of the interior vertex $\gamma$ of a closed vertex star, as this is the case of interest to us.

Corollary 3.7. Suppose $\Delta \subset \mathbb{R}^{3}$ is a vertex star with unique interior vertex $\gamma$, so the ideal associated to $\gamma$ is $\mathrm{J}(\gamma)=\left\langle\ell_{\sigma}^{r+1}: \sigma \in \Delta_{2}^{\circ}\right\rangle$. Put $\mathcal{X}=\left\{P_{\sigma}\right\}_{\sigma \in \Delta_{2}^{\circ}}$ and let $\mathrm{I}_{\mathcal{X}}=\bigcap\left\{\wp_{\sigma}: \sigma \in \Delta_{2}^{\circ}\right\} \subset \mathrm{R}$. Then

$$
\operatorname{dim} \mathrm{J}(\gamma)_{d}= \begin{cases}0 & \text { if } d \leq r \\ \operatorname{dim}\left(\frac{\mathrm{S}}{\mathrm{I}_{\mathcal{X}}^{(d-r)}}\right)_{d} & \text { if } d \geq r+1 .\end{cases}
$$

The proof of Theorem 3.6 can be found in [14], see also [15] for an introduction to inverse system of fat points and [16] for the first connection of this circle of ideas to splines.

Example 3.8. Let $\Delta$ be the regular octahedron with central vertex $\gamma$ at the origin and vertices at $( \pm 1,0,0),(0, \pm 1,0)$ and $(0,0, \pm 1)$. Then there are 12 interior two-dimensional faces which we denoted as $\sigma_{1}, \ldots, \sigma_{12}$; we number them so that they lie in the planes defined by the linear forms $\ell_{\sigma_{i}}=x$, for $i=1, \ldots, 4, \ell_{\sigma_{i}}=y$ for $i=5, \ldots 8$, and $\ell_{\sigma_{i}}=z$ for $i=9, \ldots, 12$. The dual points are $P_{\sigma_{1}}=[1: 0: 0], P_{\sigma_{5}}=[0: 1: 0]$, and $P_{\sigma_{9}}=[0: 0: 1]$, see the graph on the left of Figure 1 . These points define the ideals $\wp_{\sigma_{1}}=\langle Y, Z\rangle, \wp_{\sigma_{5}}=\langle X, Z\rangle$ and $\wp_{\sigma_{9}}=\langle X, Y\rangle$. For a positive integer $r$, and $d \geq r+1$ let $\mathrm{I}=\bigcap\left\{\wp_{\sigma}^{d-r}: \sigma \in \Delta_{2}^{\circ}\right\}$. Theorem 3.6 says that

$$
\operatorname{dim} \mathrm{J}(\gamma)_{d}=\operatorname{dim}\left\langle\ell_{\sigma}^{r+1}: \sigma \in \Delta_{2}^{\circ}\right\rangle_{d}=\operatorname{dim}(\mathrm{R} / \mathrm{I})_{d} .
$$

For example, if $r=1$ and $d=3$ then $\mathrm{I}=\langle X, Y\rangle^{2} \cap\langle X, Z\rangle^{2} \cap\langle Y, Z\rangle^{2}$ and $\operatorname{dim}(\mathrm{R} / \mathrm{I})_{3}=9$. On the other hand, $\mathrm{J}(\gamma)=\left\langle x^{2}, y^{2}, z^{2}\right\rangle$ and $\operatorname{dim} \mathrm{J}(\gamma)_{3}=9$.

Example 3.9. Let now $\Delta^{\prime}$ be a generic octahedron with central vertex $\gamma$ at the origin. Then the 12 two-dimensional faces $\sigma_{i} \in \Delta_{2}^{\prime}$ lie on 12 different planes through the origin of $\mathbb{R}^{3}$ defined by the linear forms $\ell_{i}=a_{i} x+b_{i} y+c_{i} z$. These linear forms define 12 points $\left[a_{i}: b_{i}: c_{i}\right]$ in $\mathbb{P}^{2}$. Notice that each of the edges $\tau_{j} \in \Delta_{1}^{\prime \circ}$ lies in the intersection of four of these planes, in $\mathbb{P}^{2}$ that means that the four dual points to the linear forms vanishing at those four planes lie on a line - thus there is a dotted line in Figure 1 for every interior edge of $\Delta^{\prime}$. The dual diagram in $\mathbb{P}^{2}$ is illustrated on the right in Figure 1. 

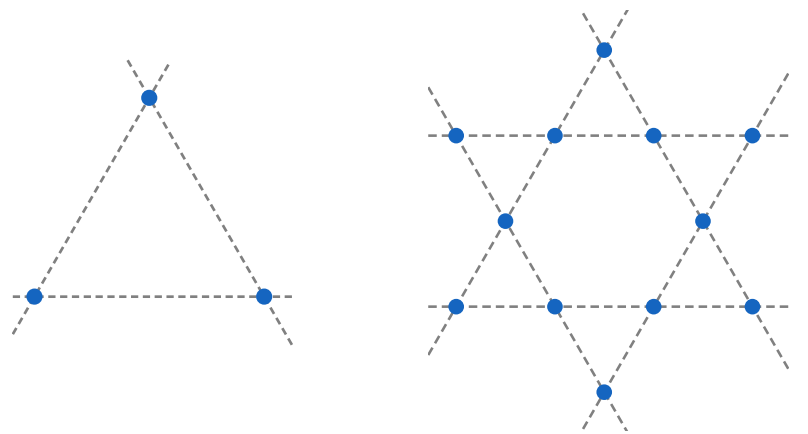

FiguRE 1. Dual graph of a regular octahedron $\Delta$ (on the left) and a generic octahedron $\Delta^{\prime}$ (on the right).

3.1. The Waldschmidt constant. Given a graded ideal $\mathrm{I} \subset \mathrm{R}$, we put $\alpha(\mathrm{I}):=\min \left\{d: \mathrm{I}_{d} \neq 0\right\}$. For instance, if $\mathcal{X}$ is a set of points in $\mathbb{P}^{2}$, then $\alpha\left(I_{\mathcal{X}}\right)$ is the minimum degree of a homogeneous polynomial which vanishes on $\mathcal{X}$. An asymptotic invariant attached to the ideal $\mathrm{I}_{\mathcal{X}}$ which has been studied in many different contexts is the Waldschmidt constant, defined as

$$
\widehat{\alpha}\left(\mathrm{I}_{\mathcal{X}}\right)=\inf _{s>0}\left\{\frac{\alpha\left(\mathrm{I}_{\mathcal{X}}^{(s)}\right)}{s}\right\} .
$$

It is known that the Waldschmidt constant is actually a limit (this follows from subadditivity of the sequence $\alpha\left(\mathrm{I}_{\mathcal{X}}^{(s)}\right)$ - see [7, Lemma 2.3.1]); so $\widehat{\alpha}\left(\mathrm{I}_{\mathcal{X}}\right)=\lim _{s \rightarrow \infty} \frac{\alpha\left(\mathrm{I}_{\mathcal{X}}^{(s)}\right)}{s}$.

Remark 3.10. The limit $\lim _{s \rightarrow \infty} \alpha\left(\mathrm{I}_{\mathcal{X}}^{(s)}\right) / s$ was first introduced by Waldschmidt [38] in complex analysis. In commutative algebra, the Waldschmidt constant gives bounds related to the containment problem; in other words for what pairs of integers $(r, s)$ we have the containment $\mathrm{I}^{(s)} \subset \mathrm{I}^{r}$ for an ideal I in a polynomial ring (see [7]).

Proposition 3.11. For a closed vertex star $\Delta \subset \mathbb{R}^{3}$, let $\Omega \subset \Delta_{2}^{\circ}$ be a finite subset of two-faces such that $\operatorname{dim} \operatorname{span}\left\{\ell_{\sigma}\right\}_{\sigma \in \Omega}=3$. Put $\mathrm{J}(\gamma)=\left\langle\ell_{\sigma}^{r+1}: \sigma \in \Omega\right\rangle, \mathcal{X}=\left\{P_{\sigma}\right\}_{\sigma \in \Omega}$, and $\mathrm{I}_{\mathcal{X}}=\bigcap\left\{\wp_{\sigma}: \sigma \in \Omega\right\}$. Then $\mathrm{J}(\gamma)_{d}=\mathrm{S}_{d} \quad$ for $\quad d>\frac{\widehat{\alpha}\left(\mathrm{I}_{\mathcal{X}}\right) r}{\widehat{\alpha}\left(\mathrm{I}_{\mathcal{X}}\right)-1}$.

Proof. By Corollary 3.7, $\mathrm{J}(\gamma)_{d}=\mathrm{S}_{d}$ if and only if $d<\alpha\left(\mathrm{I}_{\mathcal{X}}^{(d-r)}\right)$. We may assume $d>r$ (otherwise $\left.\mathrm{J}(\gamma)_{d}=0\right)$. Dividing both sides by $d-r$ gives $\mathrm{J}(\gamma)_{d}=\mathrm{S}_{d}$ if and only if

$$
\frac{d}{d-r}<\frac{\alpha\left(\mathrm{I}_{\mathcal{X}}^{(d-r)}\right)}{d-r} \text {. }
$$

Since the right hand side is larger than $\widehat{\alpha}\left(\mathrm{I}_{\mathcal{X}}\right)$, we see that

$$
\text { if } \frac{d}{d-r}<\widehat{\alpha}\left(\mathrm{I}_{\mathcal{X}}\right) \text { then } \mathrm{J}(\gamma)_{d}=\mathrm{S}_{d} \text {. }
$$

Solving for $d$ yields the proposition, provided that $\widehat{\alpha}\left(\mathrm{I}_{\mathcal{X}}\right)>1$. This latter inequality follows from a result of Chudnovsky that $\widehat{\alpha}\left(\mathrm{I}_{\mathcal{X}}\right) \geq \frac{\alpha\left(\mathrm{I}_{\mathcal{X}}\right)+1}{2}$ (see [18, Proposition 3.1]). Thus if $\alpha\left(\mathrm{I}_{\mathcal{X}}\right) \geq 2$ then $\widehat{\alpha}\left(\mathrm{I}_{\mathcal{X}}\right) \geq \frac{3}{2}>1$, so $\widehat{\alpha}\left(\mathrm{I}_{\mathcal{X}}\right)=1$ if (and only if) $\alpha\left(\mathrm{I}_{\mathcal{X}}\right)=1$, that is $\mathcal{X}=\left\{P_{\sigma}\right\}_{\sigma \in \Omega}$ is contained in a line. But this would imply that the span of the corresponding linear forms $\left\{\ell_{\sigma}\right\}_{\sigma \in \Omega}$ is at most two dimensional, contrary to assumption. 
3.2. A reduction procedure for fat points. Following the notation introduced at the beginning of Section 3, let $\Omega$ denote a collection of faces in $\Delta_{2}^{\circ}$ of a vertex star $\Delta$. The dual points defined by the linear forms vanishing on these faces define the dual points $\mathcal{X}=\left\{P_{\sigma}\right\}_{\sigma \in \Omega} \subseteq \mathbb{P}^{2}$. Consider a collection $\boldsymbol{m}$ of non-negative integers $\left\{m_{\sigma}\right\}_{\sigma \in \Omega}$, and the fat points ideal $\boldsymbol{I}_{\mathcal{X}}^{\boldsymbol{m}}=\bigcap\left\{\wp_{\sigma}^{m_{\sigma}}: \sigma \in \Omega\right\}$.

We describe the procedure introduced by Cooper, Harbourne, and Teitler in [11] to give bounds on $\operatorname{dim}\left(\mathrm{I}_{\mathcal{X}}^{\boldsymbol{m}}\right)_{d}$. The procedure uses a number of lines each of which pass through a subset of the points of $\mathcal{X}$. In our case these lines come from the edges of $\Delta$ : if $\tau \in \Delta_{1}^{\circ}$, then the pencil of linear forms vanishing on $\tau$ becomes a line in the dual space which we denote by $L_{\tau}$. In particular, the set of points $\left\{P_{\sigma}: \sigma \in \Delta_{2}^{\circ}\right.$ and $\left.\tau \subset \sigma\right\}$ all lie on $L_{\tau}$. Now suppose we are given a sequence of non-negative integers $\boldsymbol{m}$, a collection of points $\left\{P_{\sigma}\right\}_{\sigma \in \Omega}$, and the sequence of lines $L_{1}, \ldots, L_{n}$ (not necessarily different) from the collection $\left\{L_{\tau}\right\}_{\tau \in \Delta_{1}^{\circ}}$. We define the reduction vector $\boldsymbol{d}=\left(d_{1}, \ldots, d_{n}\right)$ associated to $L_{1}, \ldots, L_{n}$ inductively as follows.

(1) Starting with $L_{1}$, we define $d_{1}$ as the number of points lying on $L_{1}$, counted with multiplicity. Namely, if $L_{1}=L_{\tau}$ for some $\tau \in \Delta_{1}^{\circ}$, then $d_{1}=\sum_{\sigma \in \Omega_{\tau}} m_{\sigma}$.

(2) Reduce by 1 the multiplicities of all the points lying on $L_{1}$ and consider the sequence of points $\left\{P_{\sigma}\right\}_{\sigma \in \Omega}$ now with multiplicities $m_{\sigma}$ for $\sigma \not \ni \tau$, and $m_{\sigma}-1$ for $\sigma \ni \tau$.

(3) Repeat (1) for $L_{i}$ for $i=2, \ldots, n$ and the sequence of points with reduced multiplicities obtained in (2) at step $i-1$.

A reduction vector $\boldsymbol{d}=\left(d_{1}, \ldots, d_{n}\right)$ is said to be a full reduction vector for the fat points ideal $\mathrm{I}_{\mathcal{X}}^{\boldsymbol{m}}=\bigcap\left\{\wp_{\sigma}^{m_{\sigma}}: \sigma \in \Omega\right\}$ if $\sum_{i=1}^{n} d_{i}=\sum_{\sigma \in \Omega} m_{\sigma}$.

Example 3.12. Let $\Delta$ be the regular octahedron from Example 3.8. Taking $m_{\sigma}=2$ for every $\sigma \in \Delta_{2}^{\circ}$, and the ideal of fat points $I=\wp_{\sigma_{1}}^{2} \cap \wp_{\sigma_{5}}^{2} \cap \wp_{\sigma_{9}}^{2}$. The set of lines in this case is $\left\{L_{\tau}: \tau \in\right.$ $\left.\Delta_{1}^{\circ}\right\}=\{X, Y, Z\}$. If we take $L_{1}=Y$, two of the points lie on $L_{1}$, each of them with multiplicity 2 , so $d_{1}=4$. Notice that $\langle\mathrm{I}, Y\rangle=\left\langle X^{2} Z^{2}, Y\right\rangle$. See Figure 2, where we produce a reduction vector following starting from the dual graph of $\Delta$ in $\mathbb{P}^{2}$.
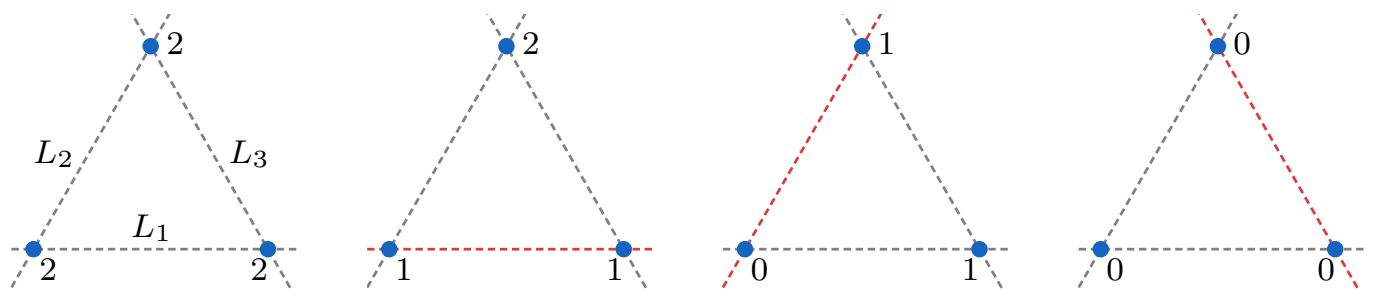

FiguRE 2. The graph of the regular octahedron $\Delta$ is composed of three points, we consider each of them to have multiplicity 2. The interior edges of $\Delta$ lie in three different lines, each of them correspond to a line in $\mathbb{P}^{2}$ as illustrated in the first graph on the left. Taking the sequence $L_{1}, L_{2}, L_{3}$, the reduction consists of the three steps (from left to right). Notice that the multiplicities are reduced to zero, and the reduction vector is $\boldsymbol{d}=(4,3,2)$.

Example 3.13. Let $\Delta^{\prime}$ be the generic octahedron from Example 3.9. Let us take $m_{\sigma}=4$ for every $\sigma \in \Delta_{2}^{\prime}$, and the ideal of fat points given by $I=\bigcap_{i=1}^{12} \wp_{\sigma_{i}}^{4}$. To construct a reduction vector associated to the ideal I, we can take any sequence of lines in $\left\{L_{\tau}: \tau \in \Delta_{1}^{\circ}\right\}$, in particular we can take a sequence so that all multiplicities reduce to zero. For instance, following the notation in Figure 3, by taking the sequence of lines $L_{1}, L_{6}, L_{4}, L_{5}, L_{3}, L_{2}$ the multiplicity at each point is reduced to 2. If we continue the reduction following the sequence of lines in the same order one more time, we get $\boldsymbol{d}=(16,16,14,14,12,12,8,8,6,6,4,4,2,2)$, and all the multiplicities are reduced to zero.

In the following theorem (and throughout this document) we define $\left(\begin{array}{l}a \\ b\end{array}\right)=0$ whenever $a<b$. 

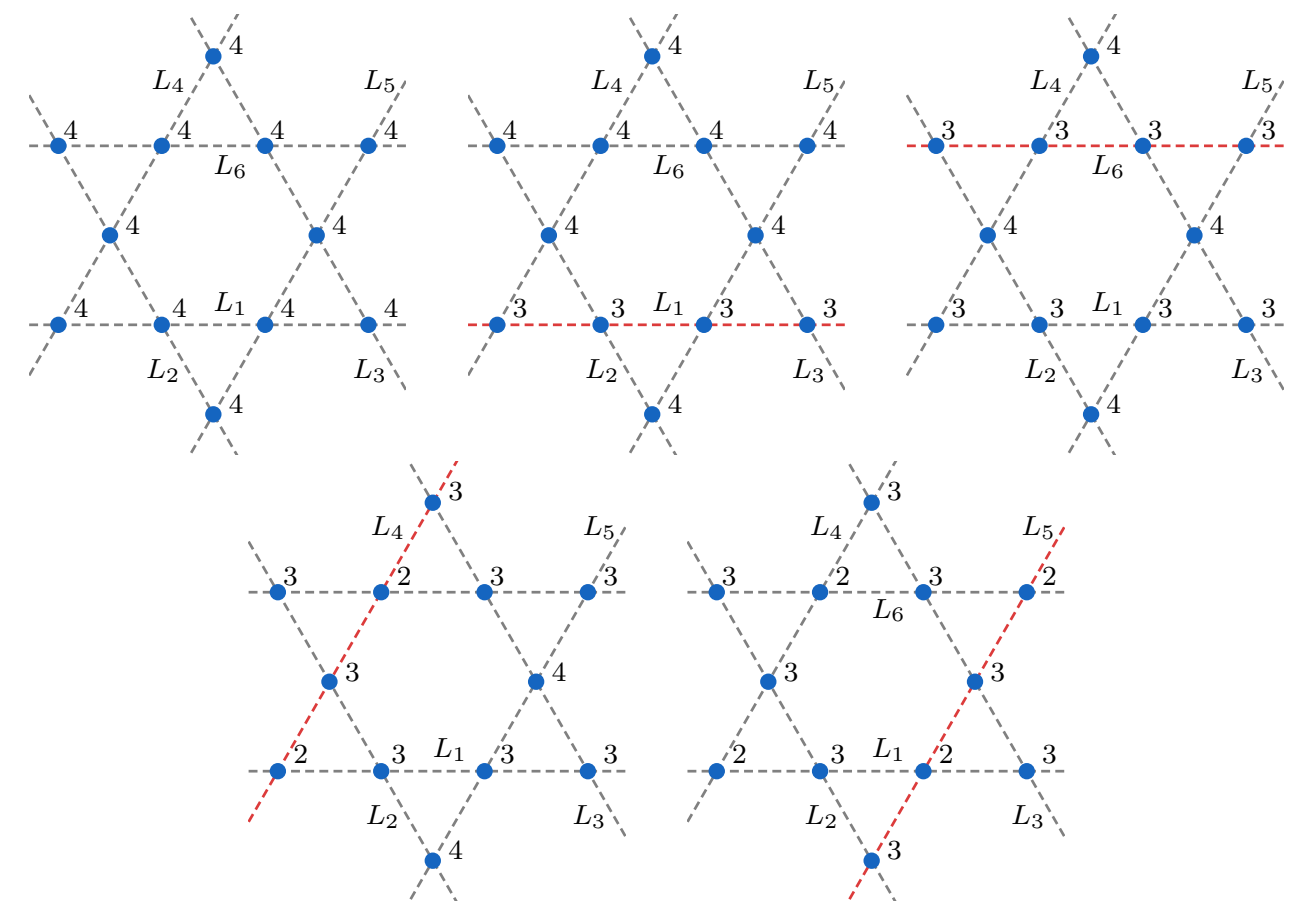

FIgURE 3. The graph of a generic octahedron $\Delta^{\prime}$ is composed by 12 points, we consider each of them to have multiplicity 4 . The interior edges of $\Delta^{\prime}$ lie on 6 different lines, each of them correspond to a line in $\mathbb{P}^{2}$ as illustrated in the first graph on the left. Taking the sequence $L_{1}, L_{6}, L_{4}, L_{5}$, the reduction consists of the four steps (from left to right). The reduction vector for this sequence of lines is $\boldsymbol{d}=(16,16,14,14)$.

Theorem 3.14. [11, Corollary 2.1.5] Let $\mathrm{I}_{\mathcal{X}}^{m}=\bigcap\left\{\wp_{\sigma}^{m_{\sigma}}: \sigma \in \Omega\right\}$ be a fat points ideal and $\boldsymbol{d}=$ $\left(d_{1}, \ldots, d_{n}\right)$ a full reduction vector from the sequence of lines $L_{1}, \ldots, L_{n}$. Then

$$
\max \left\{h_{0}^{\prime}, \ldots, h_{n}^{\prime}\right\} \leq \operatorname{dim}\left(\mathrm{I}_{\mathcal{X}}^{\boldsymbol{m}}\right)_{d} \leq\left(\begin{array}{c}
d-n+2 \\
2
\end{array}\right)+\sum_{i=0}^{n-1}\left(\begin{array}{c}
d-i-d_{i+1}+1 \\
1
\end{array}\right)
$$

where $h_{n}^{\prime}=\left(\begin{array}{c}d-n+2 \\ 2\end{array}\right)$ and $h_{i}^{\prime}=\left(\begin{array}{c}d-i+2 \\ 2\end{array}\right)-\sum_{i+1 \leq j \leq n} d_{j}$, for all $0 \leq i<n$.

If the reduction vector does not contain zeros (it is positive), the following corollary to Theorem 3.14 provides a bound on the initial degree of the ideal of fat points $I_{\mathcal{X}}^{m}$. (Crucially, for reading the following theorem, the indexing of the reduction vector was reversed between the preprint [10] and its publication [11]).

Corollary 3.15. [10, Theorem 4.2.2] If $\mathrm{I}_{\mathcal{X}}^{\boldsymbol{m}}=\bigcap\left\{\wp_{\sigma}^{m_{\sigma}}: \sigma \in \Omega\right\}$ is an ideal of fat points in $\mathbb{P}^{2}$ which has a positive full reduction vector $\boldsymbol{d}=\left(d_{1}, \ldots, d_{n}\right)$, then

$$
n+\min \left\{d_{1}-n, d_{2}-n+1, \ldots, d_{n}-1,0\right\} \leq \alpha\left(\mathrm{I}_{\mathcal{X}}^{m}\right) \leq n .
$$

Proposition 3.16. Suppose $\Delta$ is a closed vertex star with interior vertex $\gamma$ and the two-faces of $\Delta$ all span distinct planes. Let $\mathcal{X}=\left\{P_{\sigma}\right\}_{\sigma \in \Delta_{2}^{\circ}}$ be the set of points dual to the collection of forms $\left\{\ell_{\sigma}\right\}_{\sigma \in \Delta_{2}^{\circ}}$. Then $\widehat{\alpha}\left(\mathrm{I}_{\mathcal{X}}\right) \geq \min \left\{f_{1}^{\circ} / 2,3\right\}$.

Proof. Since the two-faces of $\Delta$ all span distinct planes, the set $\mathcal{X}=\left\{P_{\sigma}\right\}_{\sigma \in \Delta_{2}^{\circ}}$ of points dual to the forms $\left\{\ell_{\sigma}\right\}$ are all distinct. Choose any ordering of the interior one-faces of $\Delta$ : so $\Delta_{1}^{\circ}=\left\{\tau_{1}, \ldots, \tau_{f_{1}^{\circ}}\right\}$. 
Let $n_{i}=n_{\tau_{i}}$ be the number of faces $\sigma \in \Delta_{2}^{\circ}$ which contain $\tau_{i}$ (notice $n_{i} \geq 3$ ). Dually this gives lines $L_{1}, \ldots, L_{f_{1}^{\circ}}$ in $\mathbb{P}^{2}$ so that line $L_{i}$ contains $n_{i}$ many points of $\mathcal{X}$. Moreover, exactly two interior onefaces are contained in every interior two-face of $\Delta$. Hence each point $P_{\sigma} \in \mathcal{X}$ is at the intersection of exactly two lines from the set $\left\{L_{1}, \ldots, L_{f_{1}^{\circ}}\right\}$. See Example 3.13 for an illustration of these properties.

We define a full reduction vector $\boldsymbol{d}=\left(d_{1}, \ldots, d_{s f_{1}^{\circ}}\right)$ of length $s f_{1}^{\circ}$ for $\mathrm{I}_{\mathcal{X}}^{(2 s)}$ (where each point $P_{\sigma}$ has multiplicity $\left.2 s\right)$ as follows. This reduction vector is obtained with the sequence of lines $\left(L_{1}, L_{2}, \ldots, L_{f_{1}^{\circ}}\right)$ repeated $s$ times (in order). Since every point $P_{\sigma}$ is on exactly two of the lines from $\left\{L_{1}, \cdots, L_{f_{1}^{\circ}}\right\}$, every time the sequence $\left(L_{1}, \ldots, L_{f_{1}^{\circ}}\right)$ is completed the multiplicity of every point is reduced by two (this is why the entire reduction vector has length $s f_{1}^{\circ}$ ). On the $(k+1)$ st repetition of the sequence $\left\{L_{1}, \ldots, L_{f_{1}^{\circ}}\right\}$, the entry $d_{k f_{1}^{\circ}+i}$ (corresponding to the $(k+1)$ st time the line $L_{i}$ is repeated), satisfies

$$
d_{k f_{1}^{\circ}+i} \geq(2(s-k)-1) n_{i} \text { for } 0 \leq k \leq s-1,1 \leq i \leq f_{1}^{\circ} .
$$

This also shows that the reduction vector is positive, so we may apply Corollary 3.15. See Example 3.13, where the case $s=2$ is worked out for the generic centrally triangulated octahedron.

By Corollary 3.15, we have that $\alpha\left(\mathrm{I}_{\mathcal{X}}^{(2 s)}\right) \geq n+\min \left\{d_{1}-n, d_{2}-n+1, \ldots, d_{n}-1,0\right\}$, where $n=s f_{1}^{\circ}$. As in the previous paragraph, we consider the reduction vector indexed in the form $d_{k f_{1}^{\circ}+i}$, where $0 \leq k \leq s-1$ and $1 \leq i \leq f_{1}^{\circ}$. Now

$$
\begin{array}{rlrl}
s f_{1}^{\circ}+d_{k f_{1}^{\circ}+i}-\left(s f_{1}^{\circ}-k f_{1}^{\circ}-i+1\right) & =d_{k f_{1}^{\circ}+i}+k f_{1}^{\circ}+i-1 & \\
& \geq(2(s-k)-1) n_{i}+k f_{1}^{\circ}+i-1 & & \text { by }(4) \\
& \geq 6(s-k)-3+k f_{1}^{\circ} & & \text { since } n_{i} \geq 3, i \geq 1 \\
& =6 s-3+k\left(f_{1}^{\circ}-6\right) . & &
\end{array}
$$

So $\alpha\left(\mathrm{I}^{(2 s)}\right) \geq \min \left\{6 s-3,(s-1) f_{1}^{\circ}+3\right\}$ (the minimum depends on whether $f_{1}^{\circ} \geq 6$ or $f_{1}^{\circ}<6$ ) and

$$
\widehat{\alpha}\left(\mathrm{I}_{\mathcal{X}}\right)=\lim _{s \rightarrow \infty} \frac{\alpha\left(\mathrm{I}^{(2 s)}\right)}{2 s} \geq \lim _{s \rightarrow \infty} \frac{\min \left\{6 s-3,(s-1) f_{1}^{\circ}+3\right\}}{2 s}=\min \left\{3, f_{1}^{\circ} / 2\right\},
$$

proving the proposition.

Corollary 3.17. Suppose $\Delta$ is a closed vertex star with interior vertex $\gamma$ so that the span of every two-dimensional face is distinct. Then $\operatorname{dim} \mathrm{J}(\gamma)_{d}=\left(\begin{array}{c}d+2 \\ 2\end{array}\right)$ for $d>2 r$ if $f_{1}^{\circ}=4$, if $f_{1}^{\circ}=5$ the equality holds for $d>5 r / 3$, and if $f_{1}^{\circ} \geq 6$ then it holds for $d>3 r / 2$.

Proof. Let $\mathcal{X}=\left\{P_{\sigma}\right\}_{\sigma \in \Delta_{2}^{\circ}}$. By assumption, all the points of $\mathcal{X}$ are distinct and Proposition 3.16 applies. It follows readily from Proposition 3.11 that if $\widehat{\alpha}\left(\mathrm{I}_{X}\right) \geq M$, where $M>1$, then $\operatorname{dim} \mathrm{J}(\gamma)_{d}=$ $\left(\begin{array}{c}d+2 \\ 2\end{array}\right)$ for $d>\frac{M r}{M-1}$. Since $f_{1}^{\circ} \geq 4$, the result is immediate from Proposition 3.16.

\section{LOWER BOUND FOR SPLINES ON VERTEX STARS}

In this section we prove that the formula of [1] is a lower bound on the dimension of the homogeneous spline space on vertex stars for degrees at least $(3 r+2) / 2$, with some adjustment for four or five interior vertices according to Corollary 3.17. We will use Equation (3) from Proposition 2.6, so we first explain how to compute $\chi(\mathcal{J}, d)$ when $\Delta$ is the star of a vertex. If $\Delta$ is a closed vertex star with interior vertex $\gamma$ then the Euler characteristic of $\mathcal{J}$ has the form

$$
\chi(\mathcal{J}, d)=\sum_{\sigma \in \Delta_{2}^{\circ}} \operatorname{dim} \mathrm{J}(\sigma)_{d}-\sum_{\tau \in \Delta_{1}^{\circ}} \operatorname{dim} \mathrm{J}(\tau)_{d}+\operatorname{dim} \mathrm{J}(\gamma)_{d} .
$$

If $\Delta$ is an open vertex star, then the Euler characteristic of $\mathcal{J}$ has the form

$$
\chi(\mathcal{J}, d)=\sum_{\sigma \in \Delta_{2}^{\circ}} \operatorname{dim} \mathrm{J}(\sigma)_{d}-\sum_{\tau \in \Delta_{1}^{\circ}} \operatorname{dim} \mathrm{J}(\tau)_{d} .
$$


We use the following notation in the formulas for $\operatorname{dim} \mathrm{J}(\tau)_{d}$ and Theorem 4.7.

Notation 4.1. For a given $r \geq 0$ and $\tau \in \Delta_{1}$,

- we define $t_{\tau}=\min \left\{n_{\tau}, r+2\right\}$, where $n_{\tau}=\#\left\{\sigma \in \Delta_{2}: \tau \subset \sigma\right\}$ is the number of twodimensional faces having $\tau$ as an edge;

- and the constants

$$
q_{\tau}=\left\lfloor\frac{t_{\tau}(r+1)}{t_{\tau}-1}\right\rfloor, \quad a_{\tau}=t_{\tau}(r+1)-\left(t_{\tau}-1\right) q_{\tau}, \text { and } \quad b_{\tau}=t_{\tau}-1-a_{\tau} .
$$

Notice that $t_{\tau}(r+1)=q_{\tau}\left(t_{\tau}-1\right)+a_{\tau}$; i.e., $q_{\tau}$ and $a_{\tau}$ are, respectively, the quotient and remainder obtained when dividing $t_{\tau}(r+1)$ by $t_{\tau}-1$;

- if $\Delta$ is the star of the vertex $\gamma$ and $f_{1}^{\circ}$ is the number of interior edges in $\Delta$, we define

$$
D_{\gamma}= \begin{cases}2 r & \text { if } f_{1}^{\circ}=4 \\ \lfloor(5 r+2) / 3\rfloor & \text { if } f_{1}^{\circ}=5 \\ \lfloor(3 r+1) / 2\rfloor & \text { if } f_{1}^{\circ} \geq 6\end{cases}
$$

For the following proposition, recall that we use the convention $\left(\begin{array}{l}a \\ b\end{array}\right)=0$ when $a<b$.

Proposition 4.2. Suppose $\Delta \subset \mathbb{R}^{3}$ is the star of a vertex $\gamma, r \geq 0$ is an integer, $\sigma \in \Delta_{2}$, and $\tau \in \Delta_{1}$. Following Notation 4.1, we have

$$
\begin{gathered}
\operatorname{dim} \mathrm{S}_{d}=\left(\begin{array}{c}
d+2 \\
2
\end{array}\right) ; \quad \operatorname{dim} \mathrm{J}(\sigma)_{d}=\left(\begin{array}{c}
d+1-r \\
2
\end{array}\right) \\
\operatorname{dim} \mathrm{J}(\tau)_{d} \geq t_{\tau}\left(\begin{array}{c}
d+1-r \\
2
\end{array}\right)-a_{\tau}\left(\begin{array}{c}
d+1-q_{\tau} \\
2
\end{array}\right)-b_{\tau}\left(\begin{array}{c}
d+2-q_{\tau} \\
2
\end{array}\right)
\end{gathered}
$$$$
\operatorname{dim} \mathrm{J}(\gamma)_{d} \leq\left(\begin{array}{c}
d+2 \\
2
\end{array}\right) \text {, with equality for } d>D_{\gamma} ; \text { and }
$$

If $n_{\tau}$ is replaced by the maximum number of two-faces $\sigma$ containing $\tau$ so that $\ell_{\sigma}$ is distinct (i.e. if we set $n_{\tau}$ to be the number of distinct planes surrounding the edge $\tau$ ), then the inequality (7) is an equality. In particular, if $\Delta$ is generic with respect to $r$ and $d$ then (7) is an equality.

Remark 4.3. The formula we give for $\operatorname{dim} \mathrm{J}(\tau)_{d}$ is equivalent to those derived by Schenck [32] and Geramita and Schenck [16], although it is expressed slightly differently.

Proof. The computations for $\operatorname{dim} \mathrm{S}_{d}$ and $\operatorname{dim} \mathrm{J}(\sigma)_{d}$ are straightforward. The computation of $\operatorname{dim} \mathrm{J}(\tau)_{d}$ follows from [34] as indicated by Schenck in [32]. It also follows readily from apolarity (particularly 3.6) as shown by Geramita and Schenck in [16]. The inequality (8) follows since $\mathrm{J}(\gamma) \subset \mathrm{S}_{d}$, so $\operatorname{dim} \mathrm{J}(\gamma)_{d} \leq \operatorname{dim} \mathrm{S}_{d}=\left(\begin{array}{c}d+2 \\ 2\end{array}\right)$. The equality in (8) for $d>D_{\gamma}$ follows from Corollary 3.17. Equation (9) follows from [33, Lemma 3.2] or [28, Lemma 3.1].

If $\Delta$ is a closed vertex star we define

$$
\mathrm{LB}^{\star}(\Delta, d, r):=2\left(\begin{array}{c}
d+2 \\
2
\end{array}\right)+\left(f_{2}^{\circ}-\sum_{\tau \in \Delta_{1}^{\circ}} t_{\tau}\right)\left(\begin{array}{c}
d+1-r \\
2
\end{array}\right)+\sum_{\tau \in \Delta_{1}^{\circ}}\left(a_{\tau}\left(\begin{array}{c}
d+1-q_{\tau} \\
2
\end{array}\right)+b_{\tau}\left(\begin{array}{c}
d+2-q_{\tau} \\
2
\end{array}\right)\right) .
$$


We write $\mathrm{LB}^{\star}(d)$ instead of $\mathrm{LB}^{\star}(\Delta, d, r)$ if $\Delta, r$ are understood. If $\Delta$ is an open vertex star we define

(11)

$\operatorname{LB}^{\aleph}(\Delta, d, r):=\left(\begin{array}{c}d+2 \\ 2\end{array}\right)+\left(f_{2}^{\circ}-\sum_{\tau \in \Delta_{1}^{\circ}} t_{\tau}\right)\left(\begin{array}{c}d+1-r \\ 2\end{array}\right)+\sum_{\tau \in \Delta_{1}^{\circ}}\left(a_{\tau}\left(\begin{array}{c}d+1-q_{\tau} \\ 2\end{array}\right)+b_{\tau}\left(\begin{array}{c}d+2-q_{\tau} \\ 2\end{array}\right)\right)$.

Again we write $\operatorname{LB}^{\aleph}(d)$ if $\Delta, r$ are understood.

Proposition 4.4. Suppose $\Delta$ is a generic vertex star. If $\Delta$ is an open vertex star, then

$$
\operatorname{LB}^{\aleph}(\Delta, d, r)=\chi(\mathcal{R} / \mathcal{J}, d)=\left(\begin{array}{c}
d+2 \\
2
\end{array}\right)+\chi(\mathcal{J}, d)
$$

for every integer $d \geq 0$. If $\Delta$ is a closed vertex star with interior vertex $\gamma$ then

$$
\mathrm{LB}^{\star}(\Delta, d, r)=\left(\begin{array}{c}
d+2 \\
2
\end{array}\right)-\operatorname{dim} \mathrm{J}(\gamma)_{d}+\chi(\mathcal{R} / \mathcal{J}, d)=2\left(\begin{array}{c}
d+2 \\
2
\end{array}\right)-\operatorname{dim} \mathrm{J}(\gamma)_{d}+\chi(\mathcal{J}, d) .
$$

If $d>D_{\gamma}$ then

$$
\mathrm{LB}^{\star}(\Delta, d, r)=\chi(\mathcal{R} / \mathcal{J}, d)=\left(\begin{array}{c}
d+2 \\
2
\end{array}\right)+\chi(\mathcal{J}, d) .
$$

Proof. These follow readily from (5), (6), and Proposition 4.2.

Remark 4.5. If $\Delta$ is an open star it is well-known that $\operatorname{LB}^{\boldsymbol{\zeta}}(\Delta, d, r) \leq \operatorname{dim} \mathcal{H}_{d}^{r}(\Delta)$ (this follows from Proposition 4.4 and (9)) with equality if $d \geq 3 r+2$ and vertices are positioned generically. See [1, Theorem 3] and [34]; this essentially reduces to the planar case.

Remark 4.6. We discuss how to show that the the formulas $\operatorname{LB}^{\star}(\Delta, d, r)$ and $\operatorname{LB}^{\aleph}(\Delta, d, r)$ are the formulas appearing in Equations 15 and 16 of [1], as claimed in the introduction. We do this for $\operatorname{LB}^{\star}(\Delta, d, r)$; the computation for $\operatorname{LB}^{\aleph}(\Delta, d, r)$ is similar. If $r \in \mathbb{R}$ is any real number, put $[r]_{+}:=\max \{0, r\}$. Let $\sigma=\sum_{\tau \in \Delta_{1}} \sum_{j=1}^{d-r}\left[r+j+1-n_{\tau} j\right]_{+}$. Then we can re-write $\mathrm{LB}^{\star}(\Delta, d, r)$ as $\operatorname{LB}^{\star}(\Delta, d, r)=2\left(\begin{array}{c}d+2 \\ 2\end{array}\right)+f_{2}^{\circ}\left(\begin{array}{c}d+1-r \\ 2\end{array}\right)-f_{1}^{\circ}\left(\left(\begin{array}{c}d+2 \\ 2\end{array}\right)-\left(\begin{array}{c}r+2 \\ 2\end{array}\right)\right)+\sigma$. Using the relation $f_{2}^{\circ}=3 f_{1}^{\circ}-6$ allows us to write $\operatorname{LB}^{\star}(\Delta, d, r)$ completely in terms of $f_{1}^{\circ}$ :

$$
\mathrm{LB}^{\star}(\Delta, d, r)=(d-r)(d-2 r) f_{1}^{\circ}-2 d^{2}+6 d r-3 r^{2}+3 r+2+\sigma .
$$

If each edge is surrounded by two-faces which span distinct planes, then this is exactly the expression that appears in Equation 15 of [1]. Otherwise Equation 15 of [1] will be slightly larger. In general, Equations 15 and 16 of [1] coincide with the graded Euler characteristic $\chi(\mathcal{R} / \mathcal{J}, d)$ for $d \geq 3 r+2$.

Theorem 4.7 (Lower bound for splines on vertex stars). Let $r \geq 0, \Delta$ a closed vertex star with interior vertex $\gamma$, and $f_{1}^{\circ}$ the number of interior edges in $\Delta$. Following Notation 4.1, if $d>D_{\gamma}$ then $\operatorname{dim} \mathcal{H}_{d}^{r}(\Delta) \geq \max \left\{\left(\begin{array}{c}d+2 \\ 2\end{array}\right), \mathrm{LB}^{\star}(\Delta, d, r)\right\}$ and

$$
\operatorname{dim} \mathcal{S}_{d}^{r}(\Delta) \geq\left(\begin{array}{c}
D_{\gamma}+3 \\
3
\end{array}\right)+\sum_{i=D_{\gamma}+1}^{d} \max \left\{\left(\begin{array}{c}
i+2 \\
2
\end{array}\right), \operatorname{LB}^{\star}(\Delta, i, r)\right\}
$$

where $\operatorname{LB}^{\star}(\Delta, d, r)$ is given by the formula in (10).

Proof. We assume that $\Delta$ is a generic closed vertex star. By Proposition 2.6,

$$
\operatorname{dim} \mathcal{H}_{d}^{r}(\Delta)=\operatorname{dim} \mathrm{S}_{d}+\chi(\mathcal{J}, d)+\operatorname{dim} H_{1}(\mathcal{J})_{d}
$$

If $d>D_{\gamma}$ then by Proposition 4.4,

$$
\operatorname{dim} \mathcal{H}_{d}^{r}(\Delta)=\mathrm{LB}^{\star}(\Delta, d, r)+\operatorname{dim} H_{1}(\mathcal{J})_{d} .
$$


Thus if $d>D_{\gamma}, \operatorname{dim} \mathcal{H}_{d}^{r}(\Delta) \geq \max \left\{\left(\begin{array}{c}d+2 \\ 2\end{array}\right), \operatorname{LB}^{\star}(\Delta, d, r)\right\}$. If $\Delta$ is not generic, the conclusion follows by Lemma 2.8 .

The second statement follows since $\operatorname{dim} \mathcal{S}_{d}^{r}(\Delta)=\sum_{i=0}^{d} \operatorname{dim} \mathcal{H}_{d}^{r}(\Delta)$ and we may always take $\left(\begin{array}{c}i+2 \\ 2\end{array}\right)$ as a lower bound for $\operatorname{dim} \mathcal{H}_{i}^{r}(\Delta)$.

Remark 4.8. While it is proved in [1] that, for $d \geq 3 r+2, \operatorname{LB}^{\Uparrow}(\Delta, d, r)=\operatorname{dim} \mathcal{H}_{d}^{r}(\Delta)$ for an open vertex star and $\operatorname{LB}^{\star}(\Delta, d, r)=\mathcal{H}_{d}^{r}(\Delta)$ for a closed vertex star, the same is certainly not true for the entire spline space. The reader can see in the statement of Theorem 4.7 that if $\left(\begin{array}{c}p+2 \\ 2\end{array}\right)<\operatorname{dim} \mathcal{H}_{p}^{r}(\Delta)$ for some $p \leq D_{\gamma}$ or if $\max \left\{\left(\begin{array}{c}p+2 \\ 2\end{array}\right), \operatorname{LB}^{\star}(\Delta, p, r)\right\}<\operatorname{dim} \mathcal{H}_{p}^{r}(\Delta)$ for some $D_{\gamma} \leq p<3 r+2$, then the lower bound given for $\operatorname{dim} \mathcal{S}_{d}^{r}(\Delta)$ will be strictly less than $\operatorname{dim} \mathcal{S}_{d}^{r}(\Delta)$ in degrees $d \geq p$. Thus we cannot expect the lower bound for $\operatorname{dim} \mathcal{S}_{d}^{r}(\Delta)$ in Theorem 4.7 to give the exact dimension of the spline space in large degrees, and indeed it does not. For instance, there are special vertex positions of a centrally triangulated octahedron possessing seven linearly independent $C^{1}$ homogeneous quadratic splines, instead of the predicted six. This unexpected extra homogeneous quadratic spline appears when the three lines joining non-adjacent vertices of the octahedron all intersect in a point. This phenomenon is the three-dimensional version of the well-known planar example due to Morgan and Scott (this example was known in the 1970s [25] but does not appear in print until later - see for instance [26]). The error in predicting $\mathcal{H}_{2}^{1}(\Delta)$ for this special configuration will propagate into all degrees $d \geq 2$ for $\operatorname{dim} \mathcal{S}_{d}^{1}(\Delta)$; thus the lower bound on $\operatorname{dim} \mathcal{S}_{d}^{1}(\Delta)$ provided by Theorem 4.7 will always be off by one.

While there often exist special positions of the vertices of $\Delta$ where $\operatorname{dim} \mathcal{H}_{d}^{r}(\Delta)$ jumps, as indicated in the previous paragraph, our computations (see Tables 1 and 2) lead us to wonder if the lower bound of Theorem 4.7 for $\operatorname{dim} \mathcal{H}_{d}^{r}(\Delta)$ predicts the exact dimension for all degrees if $\Delta$ is generic. In this case the lower bound for $\operatorname{dim} \mathcal{S}_{d}^{r}(\Delta)$ would predict the correct generic dimension in all degrees. We discuss this further in Section 7.

\section{LOW DEGREE SPLINES ON GENERIC CLOSED VERTEX STARS}

This section is devoted to the proof of the following result.

Theorem 5.1 (Low degree splines on generic closed vertex stars). If $\Delta$ is a generic closed vertex star with interior vertex $\gamma$ then $\operatorname{dim} \mathcal{H}_{d}^{r}(\Delta)=\left(\begin{array}{c}d+2 \\ 2\end{array}\right)$ for $d \leq D_{\gamma}$, with $D_{\gamma}$ as in Notation 4.1.

We postpone the proof of Theorem 5.1 to introduce an auxiliary construction. If $\Delta$ is a closed tetrahedral vertex star we construct the graph of $\Delta$, denoted $G_{\Delta}$, as follows: the vertices are the interior edges, i.e. $V=\Delta_{1}^{\circ}$, and the edges correspond to the interior faces of dimension two, i.e. $E=\Delta_{2}^{\circ}$.

Remark 5.2. Suppose $\Delta$ is a closed vertex star with interior vertex $\gamma$, placed at the origin. Scaling the vertices of $\Delta$ towards or away from the interior vertex does not affect $G_{\Delta}$, so we assume all boundary vertices of $\Delta$ lie on a unit sphere centered at the origin. Then $\Delta$ is a central triangulation of the simplicial polytope formed by taking the convex hull of the vertices of $\Delta$, and $G_{\Delta}$ is the graph of this simplicial polytope [42, Chapter 3].

The following characterization of graphs of three dimensional polytopes is due to Steinitz (see [42, Chapter 4]). A graph is called $d$-connected if it remains connected after removing any set of $(d-1)$ vertices and their incident edges.

Theorem 5.3 (Steinitz). A graph $G_{\Delta}$ is the edge graph of a 3-polytope $\Delta$ if and only if it is simple, planar, and 3-connected. 
Lemma 5.4. Suppose $\Delta$ is a closed tetrahedral star. If $f_{1}^{\circ}=4$ then $G_{\Delta}$ is the complete graph on 4 vertices, so $\Delta$ is the Alfeld (or Clough-Tocher) split of a tetrahedron. If $f_{1}^{\circ}=5$ then $G_{\Delta}$ must be the graph shown on the left in Figure 4 , and $\Delta$ is the barycentric subdivision of a triangular bipyramid.
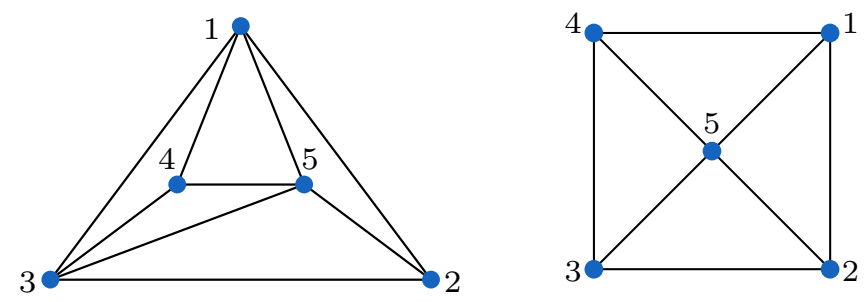

FIGURE 4. Simple 3-connected planar graphs with five vertices

Proof. If $f_{1}^{\circ}=4$ the result is clear, so we assume $f_{1}^{\circ}=5$. Euler's formula combined with the fact that each vertex must have degree at least 3 gives two possible degree sequences of simple planar 3 -connected graphs: $(3,3,4,4,4)$ or $(3,3,3,3,4)$. There is precisely one graph realizing each of these degree sequences - those pictured in Figure 4. Clearly only the one on the left is simplicial.

Proof of Theorem 5.1. The case $d \leq(3 r+1) / 2$ : As observed by Alfeld, Schumaker, and Whiteley in [4, Remark 71], this case follows from the projection techniques of Alfeld, Schumaker, and Whiteley [4] coupled with so-called vertex-splitting techniques due to Whiteley in [39].

The case $f_{1}^{\circ}=4$ and $d \leq 2 r$ : By Lemma 5.4, $\Delta$ must be the Alfeld split of a tetrahedron. It follows from a result of Schenck [29] that $\operatorname{dim} \mathcal{H}_{d}^{r}(\Delta)=\left(\begin{array}{c}d+2 \\ 2\end{array}\right)$ for $d \leq 2 r$.

The case $f_{1}^{\circ}=5$ and $d \leq(5 r+2) / 3$ : The following argument is a bit technical, and we direct the reader to [24] for unfamiliar terminology in the proof of this case. Lemma 5.4 shows that there is only one possibility for $G_{\Delta}$ (the graph on the left hand side in Figure 4). The corresponding closed tetrahedral star is a barycentric subdivision of a triangular bipyramid. Thus we show that, for generic vertex positions, the barycentric subdivision of a triangular bipyramid has no non-trivial splines in degree $d \leq(5 r+2) / 3$.

The non-trivial $C^{r}$ splines on $\Delta$ are represented as the kernel of the map

$$
\bigoplus_{\sigma \in \Delta_{2}^{\circ}} \mathrm{J}(\sigma) \stackrel{\partial_{2}}{\longrightarrow} \bigoplus_{\tau \in \Delta_{1}^{\circ}} \mathrm{J}(\tau)
$$

The graph $G_{\Delta}$ of the centrally triangulated triangular bipyramid is shown on the right in Figure 4. Orient the edge $\{i, j\}$ where $i<j$ by $i \rightarrow j$. With this choice of orientation, we can represent a tuple $G=\left(g_{\tau} \ell_{\tau}^{r+1}\right)_{\tau \in \Delta_{1}^{\circ}} \in \operatorname{ker} \partial_{2}$ by the equations

$$
\begin{aligned}
&-g_{12} \ell_{12}^{r+1}-g_{13} \ell_{13}^{r+1}-g_{14} \ell_{14}^{r+1}-g_{15} \ell_{15}^{r+1}=0 \\
& g_{12} \ell_{12}^{r+1}-g_{23} \ell_{23}^{r+1}-g_{25} \ell_{25}^{r+1}=0=0 \\
& g_{13} \ell_{13}^{r+1}+g_{23} \ell_{23}^{r+1}-g_{34} \ell_{34}^{r+1}-g_{35} \ell_{35}^{r+1}=0 \\
& g_{14} \ell_{14}^{r+1}+g_{34} \ell_{34}^{r+1}-g_{45} \ell_{45}^{r+1}=0 \\
& g_{15} \ell_{15}^{r+1}+g_{25} \ell_{15}^{r+1}+g_{35} \ell_{35}^{r+1}+g_{45} \ell_{45}^{r+1}=0
\end{aligned}
$$

The polynomials $g_{i j}$ are the smoothing cofactors of the associated spline. Suppose $G=\left(g_{\tau} \ell_{\tau}^{r+1}\right)_{\tau \in \Delta_{1}^{\circ}} \in$ ker $\partial_{2}$ is non-zero. We will show that $\operatorname{deg}(G)>(5 r+2) / 3$.

Notice first that each $g_{i j}$ appears in one of the equations (12), (13), (14), or (15). Hence if $G \neq 0$ its constituents must satisfy one of the equations (12), (13), (14), or (15) non-trivially. Suppose that $G$ only satisfies (16) trivially (i.e. $g_{15}=g_{25}=g_{35}=g_{45}=0$ ). Then $G$ must still satisfy the 
previous equations. Suppose one of $g_{12}$ or $g_{23}$ is non-zero; then by (13) $g_{12} \ell_{12}^{r+1}-g_{23} \ell_{23}^{r+1}=0$ hence both $g_{12}$ and $g_{23}$ are non-zero. Clearly in this case $g_{12}$ is a multiple of $\ell_{23}^{r+1}$ and $g_{23}$ is a multiple of $\ell_{12}^{r+1}$, hence $G$ has degree at least $2(r+1)>(5 r+2) / 3$. Likewise if one of $g_{14}$ or $g_{34}$ is non-zero then both must be and $G$ has degree at least $2(r+1)$ by (15). If $g_{12}=g_{23}=g_{14}=g_{34}=0$ in addition to $g_{15}=g_{25}=g_{35}=g_{45}=0$, then we can argue by (12) or (14) that $G$ will have degree at least $2(r+1)$ in the same way.

Now suppose that $g_{14}=g_{34}=g_{45}=0$. Then the spline $G$ restricts to a spline on the Alfeld split of a tetrahedron. As before, if $G$ is non-trivial it must have degree at least $2 r+1>(5 r+2) / 3$ by [29].

So we may assume that $G$ satisfies both (15) and (16) non-trivially. Furthermore we can assume that $g_{14}, g_{34}, g_{45}$ are all non-zero and at least two of $g_{15}, g_{25}$, and $g_{35}$ are non-zero (otherwise we could repeat the argument above, yielding that $G$ has degree at least $2(r+1)$ ). Notice that $g_{45}$ gives a non-zero element of the intersection

$$
I=\left\langle\ell_{34}^{r+1}, \ell_{14}^{r+1}\right\rangle: \ell_{45}^{r+1} \cap\left\langle\ell_{15}^{r+1}, \ell_{25}^{r+1}, \ell_{35}^{r+1}\right\rangle: \ell_{45}^{r+1},
$$

where : represents a colon ideal. That is, if $J$ is an ideal and $f$ a polynomial, $J: f$ is the ideal of all polynomials $g$ so that $f g \in J$.

We claim that this intersection is empty in degrees $d \leq(5 r+2) / 3$, which will complete the proof. To prove this claim, we make a change of variables so that $\gamma_{4}$ points along the positive $z$-axis, $\gamma_{5}$ points along the positive $x$-axis, $\gamma_{3}$ points along the positive $y$-axis, and $\gamma_{1}$ points along the ray $(t, t, t)$ where $t<0$. Under this change of coordinates, the ideal $I$ becomes

$$
I=\left\langle x^{r+1},(x-y)^{r+1}\right\rangle: y^{r+1} \cap\left\langle(y-z)^{r+1}, \ell_{25}^{r+1}, z^{r+1}\right\rangle: y^{r+1},
$$

where $\ell_{25}$ is a linear form in the variables $y$ and $z$. Put

$$
I_{1}=\left\langle x^{r+1},(x-y)^{r+1}\right\rangle: y^{r+1} \quad \text { and } \quad I_{2}=\left\langle(y-z)^{r+1}, \ell_{25}^{r+1}, z^{r+1}\right\rangle: y^{r+1} .
$$

In the rest of the proof we will show that the initial ideals in $\left(I_{1}\right)$ and in $\left(I_{2}\right)$ with respect to lexicographic order do not intersect in degrees $d \leq(5 r+2) / 3$, which will also imply that $\left(I_{1} \cap I_{2}\right)_{d}=\emptyset$.

Put $J_{1}=\left\langle x^{r+1}, y^{r+1}\right\rangle:(x+y)^{r+1} ; I_{1}$ can be obtained from $J_{1}$ by the change of coordinates $x \rightarrow x, y \rightarrow-x+y$. In [13, Lemma 7.18] it is shown that the initial ideal $\operatorname{in}\left(J_{1}\right)$ with respect to lexicographic order consists of the $\operatorname{dim}\left(J_{1}\right)_{d}$ lexicographically largest monomials in the variables $x$ and $y$. In other words, $\operatorname{in}\left(J_{1}\right)$ in lexicographic order is a so-called lex segment ideal (see [24, Chapter 2]).

We claim that $\operatorname{in}\left(I_{1}\right)$ is also a lex segment ideal. To prove this claim we consider the effect of the change of coordinates $x \rightarrow x, y \rightarrow-x+y$ on in $\left(J_{1}\right)$ in degree $d$. Under this change of coordinates, the vector space in $\left(J_{1}\right)_{d}$ becomes

$$
x^{d}, x^{d-1}(-x+y), x^{d-2}(-x+y)^{2}, \ldots, x^{d-a}(-x+y)^{a},
$$

where $a+1=\operatorname{dim}\left(I_{1}\right)_{d}$. Clearly the vector space spanned by these monomials is the same as the vector space spanned by $x^{d}, x^{d-1} y, x^{d-2} y^{2}, \ldots, x^{d-a} y^{a}$. It follows that $\operatorname{in}\left(J_{1}\right) \subset \operatorname{in}\left(I_{1}\right)$. Since $I_{1}$ and $J_{1}$ have the same Hilbert function, we must in fact have $\operatorname{in}\left(J_{1}\right)=\operatorname{in}\left(I_{1}\right)$, so $\operatorname{in}\left(I_{1}\right)$ is also a lex segment ideal.

Finally, we use some information about the Hilbert functions of $I_{1}$ and $I_{2}$. The degrees of syzygies of ideals in two variables generated by powers of linear forms are described explicitly in [32] (uniform powers) and [16] (non-uniform powers). From this analysis it follows that $\alpha\left(I_{2}\right)=\lfloor(r+1) / 3\rfloor$ (that is, the minimal generators of $I_{2}$ are in degrees at least $\left.\lfloor(r+1) / 3\rfloor\right)$. Put $K=\lfloor(r+1) / 3\rfloor$ and $N=\langle y, z\rangle^{K}$. Clearly $I_{2} \subset N$. We show that $\alpha\left(\operatorname{in}\left(I_{2}\right) \cap N\right)>(5 r+2) / 3$. 
It turns out that $I_{1}$ is a complete intersection generated in degrees $\lfloor(r+1) / 2\rfloor,\lceil(r+1) / 2\rceil$. This implies that the Hilbert function of $I_{1}$ has the following form (for a proof see [13, Corollary 7.17]):

$$
\operatorname{dim}\left(I_{1}\right)_{d}= \begin{cases}0 & \text { if } 0 \leq d \leq\lfloor(r+1) / 2\rfloor \\ 2 d+1-r & \text { if }\lfloor(r+1) / 2\rfloor \leq d<r \\ d+1 & \text { if } d \geq r\end{cases}
$$

Coupled with the fact that $\operatorname{in}\left(I_{1}\right)$ is a lex segment ideal, we obtain that a monomial $x^{a} y^{b} z^{c} \in \operatorname{in}\left(I_{2}\right)$ if and only if $2(a+b)+1-r \geq b+1$, or $2 a+b \geq r$. Similarly, $x^{a} y^{b} z^{c} \in N$ if and only if $b+c \geq\lfloor(r+1) / 3\rfloor$.

Hence to find the least degree of a monomial in $\operatorname{in}\left(I_{1}\right) \cap N$, we solve the integer linear program: minimize $a+b+c$ subject to $2 a+b \geq r$ and $b+c \geq\lfloor(r+1) / 3\rfloor$. Over the rationals, it is straightforward to check that this is minimal when $c=0, b=\lfloor(r+1) / 3\rfloor$, and $a=\frac{1}{2}(r-\lfloor(r+1) / 3\rfloor)$ with a value of $a+b+c=\frac{1}{2}\left\lfloor\frac{4 r+1}{3}\right\rfloor$. Thus $g_{45}$ must have degree greater than $\frac{1}{2}\left\lfloor\frac{4 r+1}{3}\right\rfloor$, and so $g_{45} \ell_{45}^{r+1}$ must have degree greater than $r+1+\frac{1}{2}\left\lfloor\frac{4 r+1}{3}\right\rfloor$. To prove the statement of the Proposition, it suffices to show that $\frac{5 r+2}{3}<r+1+\frac{1}{2}\left\lfloor\frac{4 r+1}{3}\right\rfloor$, which is equivalent to $\frac{4 r+1}{3}-1<\left\lfloor\frac{4 r+1}{3}\right\rfloor$. The last inequality is clearly true.

\section{ExAMPleS}

In this section we illustrate our bounds in several examples. Accompanying code can be found under the research tab at the first author's website: https://midipasq.github.io/. For the convenience of the reader we recall the notations and formulas of Section 4.

- For $\tau \in \Delta_{1}^{\circ}, n_{\tau}=\#\left\{\sigma \in \Delta_{2}: \tau \subset \sigma\right\}$ and $t_{\tau}=\min \left\{n_{\tau}, r+2\right\}$,

- For $\tau \in \Delta_{1}^{\circ}, q_{\tau}=\left\lfloor\frac{t_{\tau}(r+1)}{t_{\tau}-1}\right\rfloor, \quad a_{\tau}=t_{\tau}(r+1)-\left(t_{\tau}-1\right) q_{\tau}$, and $b_{\tau}=t_{\tau}-1-a_{\tau}$.

- If $\Delta$ is the star of the vertex $\gamma$, then $D_{\gamma}=2 r$ if $f_{1}^{\circ}=4, D_{\gamma}=\lfloor(5 r+2) / 3\rfloor$ if $f_{1}^{\circ}=5$, and otherwise $D_{\gamma}=\lfloor(3 r+1) / 2\rfloor$.

Our focus will be on closed vertex stars, so we recall the definition of $\operatorname{LB}^{\star}(\Delta, d, r)$.

$\mathrm{LB}^{\star}(\Delta, d, r):=2\left(\begin{array}{c}d+2 \\ 2\end{array}\right)+\left(f_{2}^{\circ}-\sum_{\tau \in \Delta_{1}^{\circ}} t_{\tau}\right)\left(\begin{array}{c}d+1-r \\ 2\end{array}\right)+\sum_{\tau \in \Delta_{1}^{\circ}}\left(a_{\tau}\left(\begin{array}{c}d+1-q_{\tau} \\ 2\end{array}\right)+b_{\tau}\left(\begin{array}{c}d+2-q_{\tau} \\ 2\end{array}\right)\right)$

6.1. Generic bipyramid. Let $\Delta$ be the vertex star with interior vertex $\gamma$ in Figure 5, where the vertex coordinates are chosen generically. The number of interior two-dimensional faces is $f_{2}^{\circ}=15$, and the number of interior edges is $f_{1}^{\circ}=7$. Each of the five edges in the base of the bipyramid have four two-dimensional interior faces attached to them, i.e., if we denote them by $\tau \in \Delta_{0}^{\circ}$ then $n_{\tau}=4$. The latter implies $t_{\tau}=r+2$ if $r=0,1,2$, and $t_{\tau}=4$ for every $r \geq 3$. Similarly, for the edges $\tau^{\prime}$ and $\tau^{\prime \prime}$ connecting $\gamma$ to the top and the bottom of $\Delta$, we have $n_{\tau^{\prime}}=n_{\tau^{\prime \prime}}=5$. Thus, $t_{\tau^{\prime}}=t_{\tau^{\prime \prime}}=r+2$ for $r=0,1,2$, and $t_{\tau^{\prime}}=t_{\tau^{\prime \prime}}=5$ for every $r \geq 3$.

By Theorem 4.7, $\operatorname{dim} \mathcal{H}_{d}^{r}(\Delta) \geq \max \left\{\left(\begin{array}{c}d+2 \\ 2\end{array}\right), \operatorname{LB}^{\star}(\Delta, d, r)\right\}$ for $d>D_{\gamma}$, where $D_{\gamma}=\lfloor(3 r+1) / 2\rfloor$ and

$$
\begin{aligned}
\mathrm{LB}^{\star}(\Delta, d, r)=2\left(\begin{array}{c}
d+2 \\
2
\end{array}\right)+(15- & \left.5 t_{\tau}-2 t_{\tau^{\prime}}\right)\left(\begin{array}{c}
d+1-r \\
2
\end{array}\right)+5 a_{\tau}\left(\begin{array}{c}
d+1-q_{\tau} \\
2
\end{array}\right) \\
& +5 b_{\tau}\left(\begin{array}{c}
d+2-q_{\tau} \\
2
\end{array}\right)+2 a_{\tau^{\prime}}\left(\begin{array}{c}
d+1-q_{\tau^{\prime}} \\
2
\end{array}\right)+2 b_{\tau^{\prime}}\left(\begin{array}{c}
d+2-q_{\tau^{\prime}} \\
2
\end{array}\right) .
\end{aligned}
$$



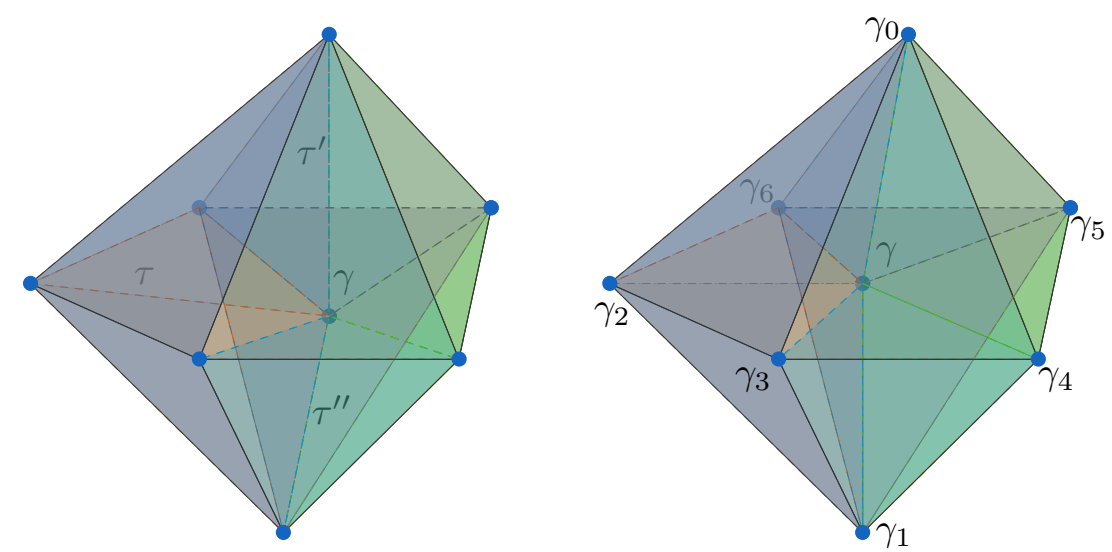

FigurE 5. Generic (left) and non-generic (right) bipyramids over a pentagon.

For instance, if $r \leq 2$, then $q_{\tau}=q_{\tau^{\prime}}=q_{\tau^{\prime \prime}}=r+2$, and therefore $a_{\tau}=a_{\tau^{\prime}}=a_{\tau^{\prime \prime}}=0$ and $b_{\tau}=b_{\tau^{\prime}}=b_{\tau^{\prime \prime}}=r+1$. Thus,

$$
\begin{aligned}
\mathrm{LB}^{\star}(\Delta, d, r) & =2\left(\begin{array}{c}
d+2 \\
2
\end{array}\right)+(15-7(r+2))\left(\begin{array}{c}
d+1-r \\
2
\end{array}\right)+7(r+1)\left(\begin{array}{c}
d-r \\
2
\end{array}\right) \\
& =5 d^{2}-15 d r+11 r^{2}+3 r+2, \text { if } d \geq r,
\end{aligned}
$$

and for $d \geq D_{\gamma}$, the lower bound on the dimension of the spline space is given by

$$
\operatorname{dim} \mathcal{S}_{d}^{r}(\Delta) \geq\left(\begin{array}{c}
D_{\gamma}+3 \\
3
\end{array}\right)+\sum_{k=D_{\gamma}+1}^{d} \max \left\{\left(\begin{array}{c}
k+2 \\
2
\end{array}\right), \mathrm{LB}^{\star}(\Delta, k, r)\right\}
$$

If $r=2$, for example, we have $D_{\gamma}=3$, and for $d \geq 3$ we get

$$
\operatorname{dim} \mathcal{S}_{d}^{2}(\Delta) \geq 20+\sum_{k=4}^{d} \mathrm{LB}^{\star}(\Delta, k, 2)=\frac{5}{3} d^{3}-\frac{25}{2} d^{2}+\frac{227}{6} d-26 .
$$

We list some numerical values of $\operatorname{LB}^{\star}(\Delta, d, r)$ in Table 1 for $1 \leq r \leq 4$ and various $d$. Table 1 also compares the values of $\operatorname{LB}^{\star}(\Delta, d, r)$ to the actual value of $\operatorname{dim} \mathcal{H}_{d}^{r}(\Delta)$ for generic vertex positions, listed under the gendim column. The value $d=D_{\gamma}$ appears in bold for each $r$. These computations were performed using the AlgebraicSplines package in Macaulay2 [17].

6.2. Non-generic bipyramid. It is possible to modify our arguments to produce better bounds in non-generic situations. We illustrate with special vertex positions for the example of the bipyramid over a pentagon - see the configuration on the right in Figure 5. Label the vertices as indicated on the right in Figure 5. We assume that $\gamma_{2}, \gamma_{3}, \gamma_{4}, \gamma_{5}$, and $\gamma_{6}$ all lie in the $x y$-plane (such configurations are studied in [9]). Assume further that $\gamma, \gamma_{0}, \gamma_{1}$, and $\gamma_{i}$ are not coplanar for $i=2, \ldots, 6$ and $\gamma, \gamma_{i}$, and $\gamma_{j}$ are not collinear for any $2 \leq i<j \leq 6$. We write $\Delta$ for this non-generic vertex star.

The collection $\mathcal{X}=\left\{\wp_{\sigma}: \sigma \in \Delta_{0}^{\circ}\right\}$ of points dual to $\left\{\sigma: \sigma \in \Delta_{0}^{\circ}\right\}$ consists of 11 points. The five two-faces with vertices $\gamma, \gamma_{i}, \gamma_{i+1}$ for $i=2,3,4,5,6$ (indices taken cyclically in this set) all span the same plane, so all correspond to the same dual point. Our assumptions for the rest of the vertices ensure that the remaining ten two-faces all span distinct planes, hence give rise to distinct dual points. Write $L_{0}, L_{1}$ for the linear forms defining the lines dual to the edges with vertices $\gamma, \gamma_{0}$ and $\gamma, \gamma_{1}$ in $\Delta$. Write $L_{i}$ for the linear form defining the line dual to the edge with vertices $\gamma, \gamma_{i}$ for $i=2, \ldots, 6$. The set $\mathcal{X}$ decomposes as a union of 5 points which lie on $L_{0}, 5$ points which lie on $L_{1}$, and the isolated point $v=[0: 0: 1]$. Since the dual points do not lie on any conic, it follows from 
Chudnovsky's bound that $\widehat{\alpha}\left(\mathrm{I}_{\mathcal{X}}\right) \geq \frac{\alpha\left(\mathrm{I}_{\mathcal{X}}\right)+1}{2}=2$. Then Proposition 3.11 implies $\operatorname{dim} \mathrm{J}(\gamma)_{d}=\left(\begin{array}{c}d+2 \\ 2\end{array}\right)$ for $d>2 r$.

Remark 6.1. A more careful analysis shows that in fact $\widehat{\alpha}\left(I_{\mathcal{X}}\right)=\frac{13}{5}$ and $\operatorname{dim} \mathrm{J}(\gamma)_{d}=\left(\begin{array}{c}d+2 \\ 2\end{array}\right)$ for $d>\frac{13}{8} r$. However we will see this more careful analysis is unnecessary.

Denote by $\operatorname{LB}_{1}^{\star}(\Delta, d, r)$ the expression which results if we replace $n_{\tau}$ by the number of distinct planes surrounding the edge $\tau$ (and thus replace $t_{\tau}$ by the minimum of $r+2$ and the number of distinct planes surrounding $\tau)$ in $\operatorname{LB}^{\star}(\Delta, d, r)$. It is shown in [1] that $\operatorname{dim} \mathcal{H}_{d}^{r}(\Delta)=\mathrm{LB}_{1}^{\star}(\Delta, d, r)$ for $d \geq 3 r+2$.

From the calculation above, $\operatorname{LB}_{1}^{\star}(\Delta, d, r)=\chi(\mathcal{R} / \mathcal{J}, d)$ for $d>2 r$, so in particular $\operatorname{LB}_{1}^{\star}(\Delta, d, r) \leq$ $\operatorname{dim} \mathcal{H}_{d}^{r}(\Delta)$ for $d>2 r$. Now put $f(d, r)=\left(\begin{array}{c}d+2 \\ 2\end{array}\right)+\left(\begin{array}{c}d+1-r \\ 2\end{array}\right)$. Since the plane $z=0$ cuts straight through $\Delta$, the spline $F$ which evaluates to $z^{r+1}$ on every upper tetrahedron and 0 on every lower tetrahedron is in $\mathcal{H}_{r+1}^{r}(\Delta)$. It follows that $f(d, r) \leq \mathcal{H}_{d}^{r}(\Delta)$ for any $d \geq 0$. Mimicking Theorem 4.7, we can take $f(d, r)$ as a lower bound on $\operatorname{dim} \mathcal{H}_{d}^{r}(\Delta)$ when $d \leq 2 r$ and $\max \left\{f(d, r), \operatorname{LB}_{1}^{\star}(\Delta, d, r)\right\}$ as the lower bound on $\operatorname{dim} \mathcal{H}_{d}^{r}(\Delta)$ when $d>2 r$. In Table 1 values of $f(d, r)$ and $\operatorname{LB}_{1}^{\star}(\Delta, d, r)$ are listed for $1 \leq r \leq 4$ and various $d$. These are compared to the actual dimension of $\mathcal{H}_{d}^{r}(\Delta)$, the values of which are in the column labeled symdim. (Again, these values were computed using the AlgebraicSplines package in Macaulay2.)

Is it possible to improve the lower bound of $\max \left\{f(d, r), \operatorname{LB}_{1}(\Delta, d, r)\right\}$ by computing $\operatorname{dim} \mathrm{J}(\gamma)_{d}$ exactly when $d \leq 2 r$ ? We show the answer is no. First of all, an application of the upper bound from [27, Theorem 4.1] (using the vertex ordering in Figure 5) shows that $\operatorname{dim} \mathcal{H}_{d}^{r}(\Delta)=f(d, r)$ for $d \leq\left\lfloor\frac{3 r+1}{2}\right\rfloor=D_{\gamma}$. This gives a range of degrees $D_{\gamma}<d \leq 2 r$ where it might be possible to improve the lower bound by using $\chi(\mathcal{R} / \mathcal{J}, d)$ instead of $f(d, r)$. Since $\chi(\mathcal{R} / \mathcal{J}, d) \leq \mathrm{LB}_{1}^{\star}(\Delta, d, r)$, if we show that $\operatorname{LB}_{1}^{\star}(\Delta, d, r) \leq f(d, r)$ for this range of values, then we have shown that we cannot improve the lower bound by using $\chi(\mathcal{R} / \mathcal{J}, d)$ for values of $d$ which are smaller than $2 r$.

In what follows we assume $r=4 k-1$ and $k \geq 1$ to simplify calculations. We compute that

$$
\mathrm{LB}_{1}^{\star}(\Delta, d, 4 k-1)=2\left(\begin{array}{c}
d+2 \\
2
\end{array}\right)-10\left(\begin{array}{c}
d+2-4 k \\
2
\end{array}\right)+10\left(\begin{array}{c}
d+2-6 k \\
2
\end{array}\right)+2\left(\begin{array}{c}
d+2-5 k \\
2
\end{array}\right) .
$$

For $d \geq 6 k-2$,

$$
\mathrm{LB}_{1}^{\star}(\Delta, d, 4 k-1)=5 d^{2}-15(4 k-1) d+200 k^{2}-90 k+10 .
$$

Also $f(d, 4 k-1)=d^{2}-(4 k-3) d+8 k^{2}-6 k+2$, for $d \geq 4 k-2$.

We can check that the polynomial $\operatorname{LB}_{1}^{\star}(\Delta, d, 4 k-1)-f(d, 4 k-1)$ attains a minimum of $-4 k^{2}-1$ at $d=7 k-3 / 2$. Furthermore the roots of $\operatorname{LB}_{1}^{\star}(\Delta, d, 4 k-1)-f(d, 4 k-1)$ are $d=7 k-3 / 2 \pm \sqrt{16 k^{2}+4}$. Thus $\operatorname{LB}_{1}^{\star}(\Delta, d, 4 k-1)<f(d, 4 k-1)$ for $6 k-2 \leq d \leq 11 k-3 / 2$. Notice this is long past the value of $d=8 k-2$ where $\operatorname{dim} \mathrm{J}(\gamma)_{d}=\left(\begin{array}{c}d+2 \\ 2\end{array}\right)$ and thus $\operatorname{LB}^{\star}(d)=\chi(\mathcal{R} / \mathcal{J}, d)$ ! So we cannot improve our lower bound by more careful computations of $\operatorname{dim} \mathrm{J}(\gamma)_{d}$ in degrees $d<2 r$. Similar arguments can be made for $r=4 k, 4 k+1,4 k+2$.

6.3. Non-simplicial vertex star. For simplicity of exposition we have only considered the case where $\Delta$ is a simplicial vertex star. However, Theorems 4.7 and 5.1 both hold verbatim if $\Delta$ is instead a polytopal vertex star. A polytopal vertex star is a collection of polytopes whose intersection contains a vertex $\gamma$ and satisfies that the intersection of each pair of polytopes is a face of both. The main difference between splines on polytopal as opposed to simplicial vertex stars is that $\operatorname{dim} H_{1}(\mathcal{J})_{d}$ may not vanish in large degree (see [23]), however this is both non-generic behavior and only makes $\operatorname{dim} \mathcal{H}_{d}^{r}(\Delta)$ larger. Thus this behavior has no impact on whether $\operatorname{LB}^{\star}(\Delta, d, r)$ is a lower bound on $\mathcal{H}_{d}^{r}(\Delta)$. 


\begin{tabular}{|c|c|c|c|c|c|c|c|}
\hline$r$ & $d$ & $\left(\begin{array}{c}d+2 \\
2\end{array}\right)$ & $\mathrm{LB}^{\star}(\Delta, d, r)$ & gendim & $\left(\begin{array}{c}d+2 \\
2\end{array}\right)+\left(\begin{array}{c}d+1-r \\
2\end{array}\right)$ & $\mathrm{LB}_{1}^{\star}(\Delta, d, r)$ & symdim \\
\hline 1 & 2 & 6 & 6 & 6 & 7 & 6 & 7 \\
\hline 1 & 3 & 10 & 16 & 16 & 13 & 16 & 16 \\
\hline 1 & 4 & 15 & 36 & 36 & 21 & 36 & 36 \\
\hline 1 & 5 & & 66 & 66 & 31 & 66 & 66 \\
\hline 1 & 6 & & 106 & 106 & 43 & 106 & 106 \\
\hline 1 & 7 & & 156 & 156 & 57 & 156 & 156 \\
\hline 1 & 8 & & 216 & 216 & 73 & 216 & 216 \\
\hline 1 & 9 & & 286 & 286 & 91 & 286 & 286 \\
\hline 2 & 3 & 10 & 7 & 10 & 11 & 12 & 11 \\
\hline 2 & 4 & 15 & 12 & 15 & 18 & 17 & 18 \\
\hline 2 & 5 & 21 & 27 & 27 & 27 & 32 & 32 \\
\hline 2 & 6 & 28 & 52 & 52 & 38 & 57 & 57 \\
\hline 2 & 7 & & 87 & 87 & 51 & 92 & 92 \\
\hline 2 & 8 & & 132 & 132 & 66 & 137 & 137 \\
\hline 2 & 9 & & 187 & 187 & 83 & 192 & 192 \\
\hline 2 & 10 & & 252 & 252 & 102 & 257 & 257 \\
\hline 2 & 11 & & 327 & 327 & 123 & 332 & 332 \\
\hline 3 & 4 & 15 & 15 & 15 & 16 & 20 & 16 \\
\hline 3 & 5 & 21 & 15 & 21 & 24 & 20 & 24 \\
\hline 3 & 6 & 28 & 25 & 28 & 34 & 30 & 34 \\
\hline 3 & 7 & 36 & 45 & 45 & 46 & 50 & 51 \\
\hline 3 & 8 & 45 & 75 & 75 & 60 & 80 & 80 \\
\hline 3 & 9 & & 115 & 115 & 76 & 120 & 120 \\
\hline 3 & 10 & & 165 & 165 & 94 & 170 & 170 \\
\hline 3 & 11 & & 225 & 225 & 114 & 230 & 230 \\
\hline 3 & 12 & & 295 & 295 & 136 & 300 & 300 \\
\hline 4 & 5 & 21 & 27 & 21 & 22 & 32 & 22 \\
\hline 4 & 6 & 28 & 22 & 28 & 31 & 32 & 31 \\
\hline 4 & 7 & 36 & 27 & 36 & 42 & 37 & 42 \\
\hline 4 & 8 & 45 & 42 & 45 & 55 & 52 & 56 \\
\hline 4 & 9 & 55 & 67 & 67 & 70 & 77 & 78 \\
\hline 4 & 10 & 66 & 102 & 102 & 87 & 112 & 112 \\
\hline 4 & 11 & & 147 & 147 & 106 & 157 & 157 \\
\hline 4 & 12 & & 202 & 202 & 127 & 212 & 212 \\
\hline 4 & 13 & & 267 & 267 & 150 & 277 & 277 \\
\hline
\end{tabular}

TABle 1. Bounds for generic and non-generic bipyramids in Sections 6.1 and 6.2 
We briefly remark on the details that need to be checked to ensure that Theorems 4.7 and 5.1 carry over to polytopal vertex stars. First, Theorem 4.7 hinges on Proposition 3.16 and Corollary 3.17. These easily carry over to polytopal vertex stars, as the simplicial nature of $\Delta$ plays no role in the proofs. Now suppose $\Delta$ is a polytopal vertex star and $\Delta^{\prime}$ is a triangulation of it which is also a simplicial vertex star (to justify the existence of such a triangulation takes a couple sentences, but it is not difficult). Then $\mathcal{H}^{r}(\Delta)$ includes into $\mathcal{H}^{r}\left(\Delta^{\prime}\right)$. By Theorem $5.1 \operatorname{dim} \mathcal{H}_{d}^{r}\left(\Delta^{\prime}\right)=\left(\begin{array}{c}d+2 \\ 2\end{array}\right)$ for $d \leq D_{\gamma}$, hence $\operatorname{dim} \mathcal{H}_{d}^{r}(\Delta)=\left(\begin{array}{c}d+2 \\ 2\end{array}\right)$ for $d \leq D_{\gamma}$ as well.

We give a simple illustration. Let $\Delta$ be the barycentric subdivision of a cube $\left(G_{\Delta}\right.$ is shown in Figure 6 ). Then $f_{2}^{\circ}=12, f_{1}^{\circ}=8$, and $n_{\tau}=3$ for every interior edge (and so $t_{\tau}=3$ if $r \geq 1$ ). We

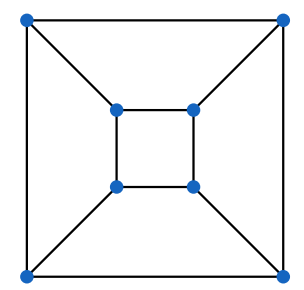

FiguRE 6. Graph of the barycentric subdivision of a cube in Section 6.3.

have

$$
\mathrm{LB}^{\star}(\Delta, d, r)=2\left(\begin{array}{c}
d+2 \\
2
\end{array}\right)-12\left(\begin{array}{c}
d+1-r \\
2
\end{array}\right)+8\left(a_{\tau}\left(\begin{array}{c}
d+1-q_{\tau} \\
2
\end{array}\right)+b_{\tau}\left(\begin{array}{c}
d+2-q_{\tau} \\
2
\end{array}\right)\right),
$$

where $q_{\tau}=\lfloor 3(r+1) / 2\rfloor, a_{\tau}$ is the remainder when $3(r+1)$ is divided by two, and $b_{\tau}=2-a_{\tau}$. If $r=2 k-1$ then

$$
\mathrm{LB}^{\star}(\Delta, d, 2 k-1)=2\left(\begin{array}{c}
d+2 \\
2
\end{array}\right)-12\left(\begin{array}{c}
d+2-2 k \\
2
\end{array}\right)+16\left(\begin{array}{c}
d+2-3 k \\
2
\end{array}\right),
$$

and if $r=2 k(k>0)$ then

$$
\mathrm{LB}^{\star}(\Delta, d, 2 k)=2\left(\begin{array}{c}
d+2 \\
2
\end{array}\right)-12\left(\begin{array}{c}
d+1-2 k \\
2
\end{array}\right)+8\left(\left(\begin{array}{c}
d-3 k \\
2
\end{array}\right)+\left(\begin{array}{c}
d+1-3 k \\
2
\end{array}\right)\right) .
$$

In Table 2 we list the values of $\operatorname{LB}^{\star}(\Delta, d, r)$ for $1 \leq r \leq 3$ and various $d$. The actual values of $\operatorname{dim} \mathcal{H}_{d}^{r}(\Delta)$ appear in the gendim column. The numbers $D_{\gamma}$ for each value of $r$ appear in bold (they are the same as in Table 1).

\section{Concluding Remarks}

In this paper we have shown that the formula of Alfeld, Neamtu, and Schumaker in [1] for homogeneous splines on closed tetrahedral vertex stars is a lower bound for $\operatorname{dim} \mathcal{H}_{d}^{r}(\Delta)$ when $d>D_{\gamma}$, and $D_{\gamma}$ is as in Notation 4.1. Using arguments due to Whiteley [39] we have also shown that, for generic vertex positions, $\mathcal{H}_{d}^{r}(\Delta)$ consists of global polynomials when $d \leq D_{\gamma}$.

Our arguments suggest that, as in the planar case, the main obstruction to computing the dimension of the spline space on a vertex star is the nontrivial homology module of the Billera-SchenckStillman chain complex. The contributions of this homology module are largely mysterious. For instance, we see from Table 1 that there are likely interesting contributions of this homology module to $\mathcal{H}_{d}^{r}(\Delta)$ for $r=3, d=7$ and $r=4, d=8,9$, where $\Delta$ is the non-generic bipyramid in Section 6.2. These contributions are 'unexpected' in the sense that we could not predict these jumps from either of the lower bounds in Section 6.2. We did not find any example of a generic closed vertex star which had similar behavior. This leads us to the following question. 


\begin{tabular}{c|c||c|c|cc|c||c|c|c}
$r$ & $d$ & $\left(\begin{array}{c}d+2 \\
2\end{array}\right)$ & $\mathrm{LB}^{\star}(\Delta, d, r)$ & gendim & $r$ & $d$ & $\left(\begin{array}{c}d+2 \\
2\end{array}\right)$ & $\mathrm{LB}^{\star}(\Delta, d, r)$ & gendim \\
\hline 1 & $\mathbf{2}$ & 6 & 0 & 6 & 2 & 9 & 55 & 62 & 62 \\
1 & 3 & 10 & 0 & 10 & 2 & 10 & & 92 & 92 \\
1 & 4 & 15 & 6 & 15 & 2 & 11 & & 128 & 128 \\
1 & 5 & 21 & 18 & 21 & 3 & $\mathbf{5}$ & 21 & 6 & 21 \\
1 & 6 & 28 & 36 & 36 & 3 & 6 & 28 & 0 & 28 \\
1 & 7 & & 60 & 60 & 3 & 7 & 36 & 0 & 36 \\
1 & 8 & & 90 & 90 & 3 & 8 & 45 & 6 & 45 \\
1 & 9 & & 126 & 126 & 3 & 9 & 55 & 18 & 55 \\
\hline 2 & $\mathbf{3}$ & 10 & 8 & 10 & 3 & 10 & 66 & 36 & 66 \\
2 & 4 & 15 & 2 & 15 & 3 & 11 & 78 & 60 & 78 \\
2 & 5 & 21 & 2 & 21 & 3 & 12 & 91 & 90 & 91 \\
2 & 6 & 28 & 8 & 28 & 3 & 13 & 105 & 126 & 126 \\
2 & 7 & 36 & 20 & 36 & 3 & 14 & & 168 & 168 \\
2 & 8 & 45 & 38 & 45 & 3 & 15 & & 216 & 216
\end{tabular}

TABLE 2. Bounds for the generic cube in Section 6.3

Question 7.1. If $\Delta$ is a generic closed vertex star and $d>D_{\gamma}$, is it true that

$$
\operatorname{dim} \mathcal{H}_{d}^{r}(\Delta)=\max \left\{\left(\begin{array}{c}
d+2 \\
2
\end{array}\right), \mathrm{LB}^{\star}(\Delta, d, r)\right\} ?
$$

Surprisingly, it seems more difficult to pose the analog of Question 7.1 for open vertex stars. In many ways, homogeneous splines on open tetrahedral vertex stars are indistinguishable from splines on planar triangulations, so we attempt to formulate Question 7.1 when $\Delta$ is a planar triangulation. In this case $\operatorname{LB}^{\aleph}(\widehat{\Delta}, d, r) \leq \operatorname{dim} \mathcal{S}_{d}^{r}(\Delta)$, where $\widehat{\Delta}$ is the open vertex star obtained by coning over $\Delta$, and $\operatorname{LB}^{\complement}(\widehat{\Delta}, d, r)$ is simply Schumaker's lower bound from [34]. One would like to ask the straightforward analog of Question 7.1: If $\Delta$ is a generic triangulation, does $\operatorname{dim} \mathcal{S}_{d}^{r}(\Delta)=$ $\max \left\{\left(\begin{array}{c}d+2 \\ 2\end{array}\right), \operatorname{LB}^{\aleph}(\widehat{\Delta}, d, r)\right\}$ ? Unfortunately there are a few sub-configurations of $\Delta$ which can force this equality to fail. We point out two of these, and would be curious to know if there are more.

First, suppose there is an interior edge in $\Delta$ both of whose vertices are on the boundary of $\Delta$; we call such an edge a chord of $\Delta$. A chord clearly gives rise to an extra spline of degree $r+1$ even for generic vertex positions. Another configuration which gives rise to splines of low degree is the following: suppose $\sigma_{1}$ and $\sigma_{2}$ are adjacent triangles of $\Delta$ with vertices $\left\{\gamma_{1}, \gamma_{2}, \gamma\right\}$ and $\left\{\gamma_{1}, \gamma_{2}, \gamma^{\prime}\right\}$, respectively. We call $\sigma_{1}, \sigma_{2}$ a boundary pair if the edges $\left\{\gamma_{1}, \gamma\right\}$ and $\left\{\gamma_{1}, \gamma^{\prime}\right\}$ are both boundary edges of $\Delta$ or the edges $\left\{\gamma_{2}, \gamma\right\}$ and $\left\{\gamma_{2}, \gamma^{\prime}\right\}$ are both boundary edges of $\Delta$. If $\sigma_{1}, \sigma_{2}$ is a boundary pair then there is a spline on $\Delta$ supported only on $\sigma_{1}$ and $\sigma_{2}$ of degree $\lfloor 3(r+1) / 2\rfloor$.

Question 7.2. If $\Delta$ is a generic triangulation without a chord or a boundary pair, does

$$
\operatorname{dim} \mathcal{S}_{d}^{r}(\Delta)=\max \left\{\left(\begin{array}{c}
d+2 \\
2
\end{array}\right), \operatorname{LB}^{\aleph}(\Delta, d, r)\right\}
$$

for every $d \geq 0$ ? If not, can the failure of equality be linked to a sub-configuration of $\Delta$ like the chord or the boundary pair?

These questions are related to Schenck's ' $2 r+1$ ' conjecture [31], which states that $\operatorname{dim} \mathcal{S}_{d}^{r}(\Delta)$ is given by Schumaker's lower bound (equivalently the graded Euler characteristic of $\mathcal{R} / \mathcal{J}$ ) for $d \geq 2 r+2$. Recently Yuan and Stillman [41] found a counterexample to this conjecture, however 
they point out that the conjecture is still open for generic triangulations. If Schenck's conjecture is true for generic triangulations, then it implies that $\operatorname{LB}^{\aleph}(\widehat{\Delta}, d, r) \geq\left(\begin{array}{c}d+2 \\ 2\end{array}\right)$ for $d \geq 2 r+2$. On the other hand, if Question 7.2 has a positive answer, then (modulo accounting for chords and boundary pairs) Schenck's conjecture for generic triangulations can be rephrased as: If $d \geq 2 r+2$, then $\operatorname{LB}^{\Uparrow}(\Delta, d, r) \geq\left(\begin{array}{c}d+2 \\ 2\end{array}\right)$. Checking this inequality simply amounts to estimating the roots of a quadratic polynomial.

\section{REFERENCES}

[1] P. Alfeld, M. Neamtu, And L. Schumaker, Dimension and local bases of homogeneous spline spaces, SIAM J. Math. Anal., 27 (1996), pp. 1482-1501, https://doi.org/10.1137/S0036141094276275.

[2] P. Alfeld And L. Schumaker, The dimension of bivariate spline spaces of smoothness $r$ for degree $d \geq 4 r+1$, Constr. Approx., 3 (1987), pp. 189-197, https://doi.org/10.1007/BF01890563.

[3] P. Alfeld And L. Schumaker, On the dimension of bivariate spline spaces of smoothness $r$ and degree $d=3 r+1$, Numer. Math., 57 (1990), pp. 651-661, https://doi.org/10.1007/BF01386434.

[4] P. Alfeld, L. Schumaker, And W. Whiteley, The generic dimension of the space of $C^{1}$ splines of degree $d \geq 8$ on tetrahedral decompositions, SIAM J. Numer. Anal., 30 (1993), pp. 889-920, https://doi.org/10.1137/ 0730047.

[5] L. BillerA, Homology of smooth splines: generic triangulations and a conjecture of Strang, Trans. Amer. Math. Soc., 310 (1988), pp. 325-340, https://doi.org/10.2307/2001125.

[6] L. Billera AND L. Rose, A dimension series for multivariate splines, Discrete Comput. Geom., 6 (1991), pp. 107-128, https://doi.org/10.1007/BF02574678.

[7] C. Bocci And B. Harbourne, Comparing powers and symbolic powers of ideals, J. Algebraic Geom., 19 (2010), pp. 399-417, https://doi.org/10.1090/S1056-3911-09-00530-X.

[8] C. Ciliberto, Geometric aspects of polynomial interpolation in more variables and of Waring's problem, in European Congress of Mathematics, vol. 201 of Progr. Math., Birkhäuser, Basel, 2001, pp. 289-316, https: //doi.org/10.1007/978-3-0348-8268-2_17.

[9] J. Colvin, D. DiMatteo, And T. Sorokina, Dimension of trivariate $C^{1}$ splines on bipyramid cells, Comput. Aided Geom. Design, 45 (2016), pp. 140-150, https://doi.org/10.1016/j.cagd.2015.12.001.

[10] S. Cooper, B. Harbourne, And Z. Teitler, Combinatorial bounds on Hilbert functions of fat points in projective space, arXiv:0912.1915v1, v1 (2009).

[11] S. Cooper, B. Harbourne, And Z. Teitler, Combinatorial bounds on Hilbert functions of fat points in projective space, J. Pure Appl. Algebra, 215 (2011), pp. 2165-2179, https://doi.org/10.1016/j.jpaa.2010. 12.006.

[12] J. Cottrell, T. Hughes, And Y. BAZILEvs, Isogeometric analysis: toward integration of CAD and FEA, John Wiley \& sons, Ltd., 2009.

[13] M. DiPasquale, Dimension of mixed splines on polytopal cells, Math. Comp., 87 (2018), pp. 905-939, https: //doi.org/10.1090/mcom/3224, https://doi.org/10.1090/mcom/3224.

[14] J. Emsalem And A. Iarrobino, Inverse system of a symbolic power. I, J. Algebra, 174 (1995), pp. 1080-1090, https://doi.org/10.1006/jabr.1995.1168.

[15] A. Geramita, Inverse systems of fat points: Waring's problem, secant varieties of Veronese varieties and parameter spaces for Gorenstein ideals, in The Curves Seminar at Queen's, Vol. X (Kingston, ON, 1995), vol. 102 of Queen's Papers in Pure and Appl. Math., Queen's Univ., Kingston, ON, 1996, pp. 2-114.

[16] A. Geramita And H. Schenck, Fat points, inverse systems, and piecewise polynomial functions, J. Algebra, 204 (1998), pp. 116-128, https://doi.org/10.1006/jabr.1997.7361.

[17] D. Grayson And M. Stillman, Macaulay2, a software system for research in algebraic geometry. Available at http://www.math.uiuc.edu/Macaulay2/.

[18] B. Harbourne and C. Huneke, Are symbolic powers highly evolved?, J. Ramanujan Math. Soc., 28A (2013), pp. $247-266$.

[19] A. Hatcher, Algebraic topology, Cambridge University Press, Cambridge, 2002.

[20] D. Hong, Spaces of bivariate spline functions over triangulation, Approx. Theory Appl., 7 (1991), pp. 56-75.

[21] A. Ibrahim And L. Schumaker, Super spline spaces of smoothness $r$ and degree $d \geq 3 r+2$, Constr. Approx., 7 (1991), pp. 401-423, https://doi.org/10.1007/BF01888166.

[22] M.-J. Lai And L. Schumaker, Spline functions on triangulations, vol. 110 of Encyclopedia of Mathematics and its Applications, Cambridge University Press, Cambridge, 2007, https://doi.org/10.1017/CB09780511721588, http://dx.doi.org/10.1017/CB09780511721588.

[23] T. McDonald And H. Schenck, Piecewise polynomials on polyhedral complexes, Adv. in Appl. Math., 42 (2009), pp. 82-93, https://doi.org/10.1016/j.aam.2008.06.001. 
[24] E. Miller And B. Sturmfels, Combinatorial commutative algebra, vol. 227 of Graduate Texts in Mathematics, Springer-Verlag, New York, 2005.

[25] J. Morgan And R. Scott, The dimension of piecewise polynomials. unpublished, 1977.

[26] J. Morgan And R. ScOTT, The dimension of the space of $C^{1}$ piecewise polynomials, (1996). unpublished, available at http://citeseerx.ist.psu.edu/viewdoc/summary?doi=10.1.1.42.4635.

[27] B. Mourrain And N. Villamizar, Bounds on the dimension of trivariate spline spaces: a homological approach, Math. Comput. Sci., 8 (2014), pp. 157-174, https://doi.org/10.1007/s11786-014-0187-8.

[28] H. Schenck, A spectral sequence for splines, Adv. in Appl. Math., 19 (1997), pp. 183-199, https://doi.org/ 10.1006/aama.1997.0534.

[29] H. Schenck, Splines on the Alfeld split of a simplex and type A root systems, J. Approx. Theory, 182 (2014), pp. 1-6, https://doi.org/10.1016/j.jat.2014.02.005.

[30] H. Schenck, Algebraic methods in approximation theory, Comput. Aided Geom. Design, 45 (2016), pp. 14-31, https://doi.org/10.1016/j.cagd.2015.11.001.

[31] H. SChenck AND P. STILleR, Cohomology vanishing and a problem in approximation theory, Manuscripta Math., 107 (2002), pp. 43-58, https://doi.org/10.1007/s002290100222.

[32] H. Schenck And M. Stillman, A family of ideals of minimal regularity and the Hilbert series of $C^{r}(\hat{\Delta})$, Adv. in Appl. Math., 19 (1997), pp. 169-182, https://doi.org/10.1006/aama.1997.0533.

[33] H. Schenck And M. Stillman, Local cohomology of bivariate splines, J. Pure Appl. Algebra, $117 / 118$ (1997), pp. 535-548, https://doi.org/10.1016/S0022-4049(97)00026-1. Algorithms for algebra (Eindhoven, 1996).

[34] L. Schumaker, On the dimension of spaces of piecewise polynomials in two variables, in Multivariate approximation theory (Proc. Conf., Math. Res. Inst., Oberwolfach, 1979), vol. 51 of Internat. Ser. Numer. Math., Birkhäuser, Basel-Boston, Mass., 1979, pp. 396-412.

[35] L. SCHUMAKER, Bounds on the dimension of spaces of multivariate piecewise polynomials, Rocky Mountain J. Math., 14 (1984), pp. 251-264, https://doi.org/10.1216/RMJ-1984-14-1-251. Surfaces (Stanford, Calif., 1982).

[36] J. ShAn, Lower bound on the dimension of trivariate splines on cells, in Approximation theory XIV: San Antonio 2013, vol. 83 of Springer Proc. Math. Stat., Springer, Cham, 2014, pp. 309-333, https://doi.org/10.1007/ 978-3-319-06404-8_18, http://dx.doi.org/10.1007/978-3-319-06404-8_18.

[37] G. Strang, Piecewise polynomials and the finite element method, Bull. Amer. Math. Soc., 79 (1973), pp. 11281137.

[38] M. WAldschmidt, Propriétés arithmétiques de fonctions de plusieurs variables. II, (1977), pp. 108-135. Lecture Notes in Math., Vol. 578.

[39] W. Whiteley, The combinatorics of bivariate splines, in Applied geometry and discrete mathematics, vol. 4 of DIMACS Ser. Discrete Math. Theoret. Comput. Sci., Amer. Math. Soc., Providence, RI, 1991, pp. 587-608.

[40] W. Whiteley, A matrix for splines, in Progress in approximation theory, Academic Press, Boston, MA, 1991, pp. $821-828$.

[41] B. Yuan and M. Stillman, A counter-example to the Schenck-Stiller " $2 r+1$ " conjecture, Adv. in Appl. Math., 110 (2019), pp. 33-41, https://doi.org/10.1016/j.aam.2019.04.004.

[42] G. Ziegler, Lectures on polytopes, vol. 152 of Graduate Texts in Mathematics, Springer-Verlag, New York, 1995, https://doi.org/10.1007/978-1-4613-8431-1.

Michael DiPasquale, Department of Mathematics, Colorado State University

Email address: michael.dipasquale@colostate.edu

$U R L:$ https://midipasq.github.io/

Nelly Villamizar, Department of Mathematics, Swansea University

Email address: n.y.villamizar@swansea.ac.uk

$U R L:$ https://sites.google.com/site/nvillami 